Man and Nature

L'homme et la nature

\title{
Approche de l'hétérologie au siècle des Lumières
}

\section{Pierre Berthiaume}

Volume 7, 1988

URI : https://id.erudit.org/iderudit/1011928ar

DOI : https://doi.org/10.7202/1011928ar

Aller au sommaire du numéro

Éditeur(s)

Canadian Society for Eighteenth-Century Studies / Société canadienne d'étude du dix-huitième siècle

\section{ISSN}

0824-3298 (imprimé)

1927-8810 (numérique)

Découvrir la revue

Citer cet article

Berthiaume, P. (1988). Approche de l'hétérologie au siècle des Lumières. Man and Nature / L'homme et la nature, 7, 79-116. https://doi.org/10.7202/1011928ar

Copyright (c) Canadian Society for Eighteenth-Century Studies / Société canadienne d'étude du dix-huitième siècle, 1988
Ce document est protégé par la loi sur le droit d'auteur. L'utilisation des services d’Érudit (y compris la reproduction) est assujettie à sa politique d'utilisation que vous pouvez consulter en ligne.

https://apropos.erudit.org/fr/usagers/politique-dutilisation/ 


\section{Approche de l'hétérologie au siècle des Lumières}

Mon opinion sur les peuples incivilisés était fixée depuis longtemps; mon voyage n'a pu que m'y affermir: "J'ai trop, à mes périls, appris à les connaître." Je suis cependant mille fois plus en colère contre les philosophes qui exaltent tant les sauvages, que contre les sauvages eux-mêmes. Ce malheureux Lamanon, qu'ils ont massacré, me disait la veille de sa mort, que ces hommes valaient mieux que nous. ${ }^{1}$

Le XVIIIe siècle s'est passionné pour cette figure de l'altérité que forme l'Amérindien, $^{2}$ mais un fait nouveau se produit: les voyageurs voient moins les Amérindiens à travers le modèle antique, comme l'a magistralement illustré Gilbert Chinard pour les XVIe et XVIle siècles, ${ }^{3}$ qu'à travers une problématique qui relève de la réflexion politique, sociale et religieuse de l'Europe des Lumières. Pour paraphraser Claude LéviStrauss, on recherche 'la société de la nature pour y méditer sur la na-

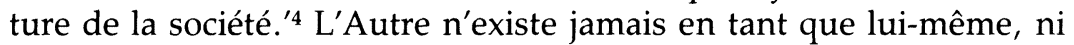
d'ailleurs en tant qu'idéal antique vivant; il sert de double et il devient l'occasion d'analyser des problèmes sous l'angle du modèle abstrait de l'homme non contaminé par les préjugés et par l'éducation. Au XVIIIe siècle, le recours à la figure de l'Autre est d'abord polémique et comme le rappelle de façon imagée Hélène Clastres, si 'le cannibale de Montaigne fait de l'ethnologie,' les 'sauvages de Diderot ou de La Hontan font plutôt de la morale. ${ }^{\prime 5}$ À la limite, le 'Sauvage' n'est rien de plus qu'une commode catégorie épistémologique à usage européen. On ne peut que souscrire aux thèses de Michèle Duchet qui observe qu'au XVIIIe siècle,

s'estompent les caractères originaux d'une humanité, dont on ne retient que les traits susceptibles de fournir un modèle, ou à l'inverse de dénoncer l'illusion d'un modèle. La réalité du monde sauvage demeure enserrée dans un réseau de négations, qui, par le jeu des combinaisons, permet la construction de modèles antithétiques. Tantôt il est question de peuples sans histoire, sans écriture, sans religion, sans moeurs, sans police, et dans ce premier type de 
discours les négations se combinent avec des traits marqués positivement pour signifier le manque, le vide immense de la sauvagerie opposé au monde plein du civilisé. Tantôt on envie ces mêmes peuples qui vivent sans maîtres, sans prêtres, sans lois, sans vices, ni tien ni mien, et les négations, combinées ici avec des traits marqués négativement, disent le désenchantement de l'homme social et l'infini bonheur de l'homme naturel. Dans le premier cas, le parallèle tourne à l'avantage de l'homme policé, dans le second, la différence est tout entière au désavantage de l'homme social. Il s'ensuit qu'un seul changement de signe suffit à inverser tout le sens du discours: de Voltaire à Rousseau ou Diderot, ce sont moins ses éléments qui varient que leur distribution dans un système où ils sont affectés tantôt du signe plus, tantôt du signe moins. ${ }^{6}$

Tantôt modèle, tantôt repoussoir, l'Autre n'est jamais véritablement considéré en lui-même: toujours le discours le récupère et fait de sa rencontre une occasion de réflexion sur l'homme moderne. En ce sens, l'Autre subit le traitement qu'Hérodote, déjà, lui faisait subir: essentiellement, il s'agit de traduire l'Autre, de l'expliquer de façon à 'faire passer l'Autre au même. ${ }^{17}$ Michèle Duchet:

ni les anciens historiens ni les premiers explorateurs de l'intérieur de l'Afrique ou du continent américain ne se donnèrent pour but d'observer et de décrire les sociétés avec lesquelles ils entrèrent en contact en faisant abstraction de leur propre société, de leurs habitudes ou de leurs préjugés. Loin d'être objet de connaissance, le monde sauvage n'existe pour eux qu'à travers une certaine pratique, qui leur interdit de renoncer à leur statut de civilisé pour n'être que des observateurs-participants, à la manière des ethnographes modernes. ${ }^{8}$

$\mathrm{Au}$ XVIIIle siècle, les voyageurs n'adoptent pas une attitude différente et ils ne s'intéressent à l'Autre que pour y déchiffrer des signes à traduire en discours. C'est ainsi qu'au cours du siècle et en fonction des nécessités intellectuelles le 'Sauvage' se transforme en 'bon Sauvage,' puis qu'il se métamorphose en concept, enfin en mythe, avant de céder la place à une autre figure signifiante, I'Insurgent' américain. C'est cette hétérologie qu'il faut interroger. ${ }^{9}$ L'itinéraire du baron de La Hontan servira d'amorce à la réflexion.

Dans les Nouveaux Voyages de Mr Le Baron de La Hontan dans l'Amerique septentrionale, l'Amérindien apparait d'abord comme objet d'un spectacle destiné au regard du voyageur et du lecteur et qui prend son sens en eux, par exemple lorsque La Hontan rappelle l'attitude des Iroquois qui 'se moquent des menaces [du] Roi \& [des] Gouverneurs, ne connoissant en aucune maniere le terme de dépendance, ${ }^{\prime 10}$ ou encore lorsqu'il montre des 'Sauvages' prêts à se convertir pour 'attraper quelque pipe de Tabac.'11 Dans les deux cas, La Hontan se sert des Amérin- 
diens pour ridiculiser administrateurs et missionnaires, et plus largement, pour critiquer l'ensemble de la politique coloniale et l'idée même d'évangélisation. Il lui suffit de montrer la conduite de vrais Hurons et de vrais Iroquois, de la donner en spectacle, pour jeter du discrédit sur les valeurs que véhiculent la société et la foi. Mais c'est bel et bien une mise en scène que réalise alors La Hontan. Non content d'agiter des marionnettes sur son théâtre de foire, en bon pédagogue, il souligne et il explicite parfois le sens des discours, mieux, il commente le déroulement des scènes et il n'hésite pas à glisser quelques réflexions de son cru au cours de la représentation pour en assurer l'intelligence. C'est que l'Autre, le Sauvage, paraît d'abord en contraste par rapport à l'homme policé et civilisé et que sa différence, pétrie de naïveté, sinon d'infantilisme intellectuel, fait de lui un être sur lequel on peut lire en transparence et sans difficulté les étapes de l'aliénation, religieuse d'abord, sociale ensuite. ${ }^{12} C^{\prime}$ est cette lecture que pratique La Hontan quand il se sert du témoignage de l'Autre pour extrapoler et pour généraliser des critiques qui sont en fait les siennes:

Je suis maintenant convaincu que la Tradition est trop suspecte, inconstante, obscure, incertaine, trompeuse \& vague, pour se fier à elle: J'ai l'obligation de cette idée aux Sauvages du Canada, qui ne sçachant rapporter au vrai ce qui s'est passé dans leur Païs il y a deux cens ans, me font révoquer en doute la pureté \& l'incorruptibilité de la Tradition. ${ }^{13}$

Pour reprendre le paradoxe évoqué dans l'Histoire philosophique et politique des Etablissemens $\mathcal{E}$ du Commerce des Européens dans les deux Indes, c'est 'l'ignorance des Sauvages qui a éclairé les peuples policés. ${ }^{14} \mathrm{En}$ fait, c'est de l'abaissement même du Sauvage que La Hontan tire des leçons applicables à l'ensemble du genre humain. L'Autre est d'abord dans les Nouveaux Voyages un exemple bien concret qui inspire au philosophe des réflexions, voire un témoin qui illustre l'involution des hommes lorsqu'ils sont soumis aux superstitions religieuses et qu'ils s'abâtardissent au sein des sociétés. Au départ, comme objet soumis au regard et à l'analyse, l'Autre est rien moins que sujet: au contraire, exemplum vivant d'un regrès, il est avant tout, malgré sa résistance à l'avancée coloniale, un être en train de se perdre et qui appelle la réflexion du voyageur. Aussi devient-il un double: le double négatif du philosophe, le creux dans lequel se sont logés tous les mensonges et toutes les fausses illusions imposés par la Tradition et par ses mages. Mais foin alors du mythe antique. Seul demeure un être frustre, pour tout dire un 'Sauvage' qui se donne d'autant plus facilement à lire qu'il est sans fard pour camoufler sa disgrâce.

Encore faut-il que l'Amérindien puisse prendre la parole pour dénoncer les causes de son aliénation et partant, défendre des valeurs 
positives. Alors seulement peut s'engager un 'dialogue.' En fait, cette métamorphose du Sauvage en philosophe relève de procédés littéraires. Dans ses Nouveaux Voyages, La Hontan narre une dispute qu'il aurait eue avec un médecin portugais au sujet de l'origine des Amérindiens. La scène a lieu dans une auberge et les propos échangés opposent le principe de la monogénésie, en accord avec les textes sacrés, à celui d'une polygénésie, rejeté par l'Eglise. Or dans l'échange, La Hontan assume le rôle du défenseur de la Tradition, mais si maladroitement qu'il la caricature. Son argumentation ne convainc personne et ses protestations sonnent faux. C'est son adversaire qui reste maître $\mathrm{du}$ terrain et le dialogue formule très nettement une critique des positions arrêtées de l'Eglise. Bien sûr le procédé est rhétorique et des plus classiques. Gustave Lanson observe qu'il est fréquemment pratiqué à la fin du XVIIe siècle et au début du XVIIIe, notamment par Fontenelle dans ses Entretiens sur la pluralité des mondes (1686), par Aubert de Versé, soupçonné de faire paraître un anti-Spinoza, L'impie convaincu (1685), pour mieux exposer la doctrine du philosophe, et par Boulainvilliers qui publie une (fausse) Réfutation de Spinoza en $1731 .{ }^{15}$ Quoi qu'il en soit, le procédé des Nouveaux Voyages annonce les Dialogues. ${ }^{16}$ Il suffira de remplacer l'interlocuteur portugais, vrai ou fictif, par un Amérindien, et qui mieux est par un personnage historique, et de 'raisonner avec' lui pour formuler une critique radicale de l'ensemble des valeurs sur lesquelles repose la société européenne. ${ }^{17}$ Adario, vague croisement du Huron Kondiaronk, ce 'Machiavel né dans les forêts' au dire de l'abbé Raynal, et de La Hontan lui-même, ${ }^{18}$ assume le rôle de philosophe rustique pour mieux dénoncer les 'sottises' de la société blanche. ${ }^{19}$ L'artifice est le même, y compris les maladresses du narrateur, toujours à court d'arguments. ${ }^{20}$ En fait, il se résume, de façon bien classique depuis Platon, à dédoubler une analyse en argumentation entre deux protagonistes et à utiliser l'un comme porte-parole des thèses avancées, l'autre comme instrument pour relancer la discussion et comme faire-valoir du premier. C'est alors que le 'Sauvage,' parce que demeuré plus près de la nature et partant, moins corrompu en apparence par les sophismes de la société, devient porteur des revendications de la frange la plus éclairée des milieux intellectuels de la fin du XVIIe siècle. Mais La Hontan s'inspire alors des règles du roman utopique et d'un genre promis à un bel avenir, le roman qui donne la parole à des étrangers pour observer et pour juger la société française. Au premier, il emprunte la technique du dialogue entre le voyageur qui aborde en 'utopie' et un 'Ancien' de celle-ci qui permet $\mathrm{d}^{\prime}$ 'exposer de nouvelles règles sociales et par contraste de condamner celles de l'Europe. ${ }^{21} \mathrm{Au}$ second, il emprunte l'idée de faire juger la société française par un étranger. Cette fois, c'est vraisemblablement 
l'ouvrage de Giovanni-Paolo Marana, l'Espion du Grand-Seigneur, publié en 1684, qui fournit le modèle. ${ }^{22}$ Comme Mamut, le Turc bien italien de Marana, Adario promène sur la société un faux regard ingénu, d'autant plus sévère qu'il s'appuie sur un esprit critique qui perçoit la société non plus de l'intérieur et comme allant de soi, mais de l'extérieur, en étranger qui observe tout d'un oeil neuf. Chez La Hontan, c'est la différence relative qui caractérise l'Amérindien qui permet une comparaison constante entre le monde sauvage et le monde européen au détriment du second. En somme, c'est sa singularité qui lui donne prise sur la société française. ${ }^{23}$

Du reste, entre les Nouveaux Voyages et les Dialogues, le personnage du Sauvage philosophe évolue. En 1703, la parole de l'Autre paraît encore bien timide et elle se cantonne dans des 'harangues,' le plus souvent de circonstance, entre autres lorsque 'Grangula' dénonce l'incurie de La Barre. ${ }^{24}$ Mais l'exemple est d'autant plus intéressant que la gestuelle caractéristique du cérémonial amérindien et l'intelligence du discours mettent déjà le Sauvage en vedette. ${ }^{25}$ En fait, les rôles sont inversés: c'est l'Amérindien qui parle et c'est La Barre qui est réduit au rang d'auditeur d'autant plus passif que flanqué de troupes peu aguerries et qui pis est, décimées, il ne peut pas s'imposer et qu'il doit supporter le discours de l'adversaire. À tout prendre, les Dialogues ne font que reprendre le scénario avec de nouveaux personnages et en l'amplifiant: même impuissance du Blanc à s'imposer, même autorité de l'Amérindien. Mais cette fois, ce sont des questions plus générales qui sont abordées et elles le sont plus longuement. Aussi, paradoxalement, au moment où l'Autre devient véritablement sujet du discours, qu'il dialogue et qu'il raisonne, il défend moins les intérêts objectifs des siens qu'il ne propose une réflexion sur les valeurs de la civilisation occidentale. ${ }^{26}$ En somme, il prend la parole pour parler 'philosophe' et il devient un 'bon Sauvage,' ou selon la terminologie anglaise, plus révélatrice encore, un 'noble Sauvage,' c'est-à-dire, une figure qui agit comme révélateur des failles de la société, finalement un personnage fictif dans lequel René Gonnard reconnaît la projection d'un 'idéal d'affranchissement complet à l'égard de toute loi morale, religieuse, sociale, de toute Obligation,' ce qui l'amène à réduire la figure de l'Autre à un phénomène d'ordre psychologique. ${ }^{27}$ Or la réalité paraît plus complexe, surtout que la figure du 'bon Sauvage' est déjà marquée par une longue tradition. ${ }^{28}$

Mais commençons par circonscrire le personnage, ses assises épistémologiques et son rôle, surtout que dans le cas précis des relations de voyages, le modèle est soumis aux pressions contradictoires de l'observation sur le terrain et des contraintes polémiques impliquées par l'usage philosophique qu'on en veut faire, et que l'utilisation de 
l'Autre comme miroir révélateur des tares de l'homme policé n'est pas neuve et qu'elle obéit à des clichés. Sans citer les nombreuses Relations des Jésuites qui illustrent la conduite des Hurons ou des Iroquois pour mieux dénoncer celle des chrétiens, ${ }^{29}$ on peut rappeler la réflexion de Gabriel Sagard en 1632 lorsqu'il évoque l"humanité' des Hurons qui l'ont accueilli, ce qui le 'faisoit grandement rentrer en (lui) mesme, \& admirer leur constance, \& le pouuoir qu'ils ont sur leurs propres passions, \& comme ils sçauent bien se supporter les vns les autres, \& s'entresecourir \& assister au besoin. ${ }^{30}$ Toutefois ces réflexions restent toujours subordonnées aux certitudes de la religion. Or, dans une large mesure, l'Histoire generale des Isles de S. Christophe, de la Gvadelovpe, de la Martinique et avtres dans l'Amerique de Jean-Baptiste du Tertre (1654) permet de dépasser cette problématique en dichotomisant l'Amérindien. Pour du Tertre, imbu de botanique, le 'Sauvage' se compare à la plante sauvage, c'est-à-dire aux 'plantes' et aux

fruicts que la nature produit sans aucune culture dans les forests \& dans les deserts, lesquelles quoyque nous appellions Sauuages, possedent pourtant les vrayes vertus \& les proprietez dans leur force \& dans leur entiere vigueur, lesquelles bien souuent nous corrompons par nos artifices, \& alterons beaucoup, lorsque, nous les plantons dans nos jardins. ${ }^{31}$

Par analogie, le Sauvage devient cet être naturel qui n'a pas subi les fâcheuses influences de l'artifice et qui demeure une créature saine:

il est à propos de faire voir dans cette cinquiéme Partie que les Sauuages de ces isles sont les plus contens, les plus heureux, les moins vicieux, les plus sociables, les moins contrefaits, \& les moins tourmentez de maladies, de toutes les nations du monde. Car ils sont tels que la nature les a produit, c'est à dire dans vne grand simplicité \& naïueté naturelle: ils sont tous égaux, sans aucune sorte de superiorité ny de seruitude; \& à peine peut-on reconnoistre aucune sorte de respect mesme entre les parens, comme du fils au pere. Nul n'est plus riche, ny plus pauure que son compagnon, \& tous vnanimement bornent leur désirs à ce qui leur est vtile, \& precisément necessaire, \& méprisent tout ce qu'ils ont de superflu, comme chose indigne d'estre possedée. ${ }^{32}$

Certes il leur manque la grâce, mais précisément la grâce paraît être un ajout, un supplément, sans doute indispensable aux yeux du prêtre, mais dont l'absence n'enlève rien au portrait positif de l'homme naturel. ${ }^{23} \mathrm{Au}$ total, chez l'Amérindien, on trouve un homme, éminemment exemplaire, et un païen qu'il s'agit de convertir. Or il suffira d'ignorer ce second aspect du personnage, ou de l'occulter, pour rendre 
la figure de l'Autre opératoire sur le plan philosophique. En laissant tout simplement de côté l'une des deux caractéristiques de l'Amérindien, tel que défini par les missionnaires, en passant sous silence la question de la rédemption, on transforme l'Autre en 'philosophe rustique.' De là une figure qui est loin de posséder les contours nets qu'on prête trop souvent au 'bon Sauvage': si c'est à la condition d'être libéré de l'hypothèse métaphysique ou de l'inverser que l'Amérindien peut engager le dialogue, c'est aussi en se rattachant à une tradition édificatrice propre aux relations missionnaires qu'il le fait.

En réalité, la question de l'Autre s'inscrit alors non seulement à l'intérieur d'une problématique moralisante (l'a-corruption de l'homme naturel qui sert de miroir à l'occidental christianisé et policé), mais à l'intérieur d'une ontologie qui conçoit l'homme en marge de la nature. En effet, déjà dans la perspective chrétienne, l'homme

n'est pas situé dans la nature comme un élément dans un ensemble; il n'a pas sa place en elle comme les choses ont leur place; il est transcendant au monde physique; il n'appartient pas à la nature mais à la grâce, qui est surnaturelle. ${ }^{34}$

Toute la tradition du christianisme fait de l'homme un être élu parmi les créatures, et partant une singularité au sein de la nature. L'abandon (tout relatif souvent) du christianisme par les 'philosophes' ne change rien au problème quand il n'en accentue pas le caractère dramatique. Une fois l'hypothèse métaphysique levée à travers le matérialisme sensualiste des penseurs de la fin du XVIIe siècle, l'homme se retrouve plus démuni que jamais pour rendre compte de son existence et de la place qu'il occupe au sein de l'univers. Au moins dans le christianisme l'homme trouvait-il un sens à sa présence au monde dans la surnature dont il était le dépositaire, surtout que toute la création n'avait de cesse de témoigner de la divine Providence et de l'existence même de Dieu. Mais une fois disparu Dieu, une fois évanouie l'idée que l'univers n'est que l'antichambre d'une plénitude à venir, l'homme se retrouve seul face à l'infini, ou plutôt face au néant. Ainsi sa place au sein de la nature pose-t-elle davantage problème chez les philosophes, qu'ils soient croyants du reste, que chez les théologiens: que ce soit chez Spinoza pour qui l'homme apparaît comme un mode fini d'existence au sein d'une nature infinie qui devient par là la réalisation concrète de l'essence de la Divinité, ou que ce soit chez René Descartes qui fonde sa philosophie sur l'impossible union de l'âme et de la pensée avec la matière, toujours l'homme apparait comme une singularité et comme un être corruptible en rupture de ban avec la nature. C'est qu'à la fin du XVIIe siècle, la philosophie découvre que rien de ce qui paraît 'naturel' ne peut justifier la singularité de l'homme au sein de 
la création: l'essence de l'homme, ce qui fait sa spécificité et ce qui le distingue des autres créatures, notamment la pensée, le langage et sa grégarité ordonnée en société, ne paraît reposer que sur des ajouts à la nature. Au XVIIIe siècle, on conçoit 'l'ordre des différences qui, en dépit de tout, sépare l'homme de l'animal ... comme celui d'un écart, d'un ajout à la nature animale de l'homme. ${ }^{35}$ L'opposition entre nature et culture s'exprime alors en termes d'aliénation, l'homme ayant dû finalement trahir sa nature pour se policer: en effet, le fait culturel

est réduit à l'assomption d'un ensemble limité de Règles, destinées à assurer la subsistance et la survie de l'espèce - Règles par essence arbitraires puisque inexistantes dans la nature et par ailleurs totalement irrévocables. Défini de cette façon, comme un ordre nouveau coupé de la nature, quoique motivé par elle, et d'une nature qui est donnée comme insuffisante voire mauvaise, le concept de culture ne pouvait que mener au désarroi et au vertige; l'homme semblait exclu des plans de la nature et le régime qu'il s'était construit était au sens strict, celui de l'aliénation. ${ }^{36}$

En fait, aux yeux de Thomas Hobbes, de Spinoza et des nombreux juristes qui analysent les fondements sur lesquels repose la société, pour survivre, l'homme a dû abandonner un mode de vie sylvestre qui faisait de lui la proie des autres animaux et il a dû inventer le langage et la société pour éviter d'être éliminé de la surface de la terre. C'est pourquoi, par définition, toute société est a-naturelle car issue de conventions que les hommes ont dû passer entre eux pour assurer la survie de l'espèce. De plus, ces conventions doivent paradoxalement être à la fois arbitraires (en tant que conventions) et universelles (on ne saurait concevoir l'existence d'une société sans elles). C'est à l'intérieur de cette fracture entre nature et culture que se situe surtout la question de l'Autre au XVIIIe siècle, non pas tant parce que le Sauvage représente l'homme de l'anomie, l'homme d'avant le contrat, mais par ce qu'il témoigne de conventions plus proches des lois naturelles, plus respectueuses des lois inscrites dans la nature. Entre l'état policé et l'état de nature qu'un Locke et qu'un Rousseau définissent de façon absolument abstraite, trouve place un état sauvage qui autorise le questionnement de la société. C'est sur ces prémisses philosophiques que repose toute représentation du Sauvage qui emprunte aussi aux procédés rhétoriques et littéraires comme on vient de le voir chez La Hon$\tan .{ }^{27}$ D'abord figure, celle du 'bon Sauvage,' au nom des principes éthiques, l'Autre passera à l'état de concept au moment où au lieu de se contenter de dénoncer les tares de la société, on interrogera ses fondements. 
La figure d'abord. Le XVIIIe siècle exploitera l'idée à satiété, y compris les voyageurs, et en 1709, Gautier du Tronchoy observe au sujet des naturels des 'Isles' qu'il

n'y a point d'hommes sur la terre qui vivent plus contens \& plus heureux, \& qui soient moins vicieux, moins contrefaits, moins tourmentez de maladies de toutes les nations du monde \& plus sociables; ils sont tels que la nature les a produit [sic], c'est-à-dire, dans une grande simplicité \& naïveté naturelle. ${ }^{38}$

En outre, ils 'ne sçavent guere de malice que ce que nos François leur en apprenne' (sic). ${ }^{39}$ Le mal ne provient que de la civilisation et l'opposition entre la nature, essentiellement bonne, et la culture, dont le propre est de pervertir, se réalise intégralement ici. Et pourtant, des observations montrent que cette pure nature ne satisfait pas complètement Gautier du Tronchoy. ${ }^{40}$ En fait, tout se passe comme si les voyageurs esquissaient une image positive des Amérindiens moins en fonction de la réalité qu'ils ont sous les yeux qu'en fonction de la volonté de prendre le contre-pied de la civilisation, perçue comme corruptrice et aliénante. On conçoit que l'Amérindien, membre d'une société en apparence dénuée de règles et d'arbitraire culturel aux yeux des philosophes, soit devenu un modèle épistémologique par le biais de la figure du 'bon Sauvage' dont la fonction se réduit à démasquer la corruption du monde policé et dénaturé, et qui se caractérise par l'inversion: essentiellement, l'Autre forme un envers, la négation vivante de l'Européen et de son monde, et il se définit par opposition, par ce qu'il n'est pas. Il est d'abord celui qui ne reconnait 'ni Tien ni Mien, ${ }^{\prime 41}$ en somme, celui qui nie la notion de propriété, à l'origine de tous les maux de l'humanité ${ }^{42}$ Toutefois, à partir de là, par le biais d'une suite de déductions qui empruntent peu à l'observation, l'Autre revêt des caractéristiques qui en font un être contestatire par la liberté qu'il trouve, curieusement, à se soumettre aux impératifs d'une nature identifiée à la raison: 'les loix,' de demander Adario, 'n'est ce pas dire les choses justes \& raisonnables? Tu dis qu'oüy; \& bien, observer les Loix, c'est donc observer les choses justes \& raisonnables. ${ }^{43}$ Aussi, le Huron tiret-il la conséquence logique de son assertion en opposant les Amérindiens qui vivent 'simplement sous les Loix de l'instinct, \& de la conduite innocente que la Nature sage [leur] a imprimés dès le berceau, ${ }^{\prime 44}$ aux Européens en général et aux Français en particulier qui se soumettent aux règles factices de la bienséance, qui règlent leur conduite sur l'apparence de la vérité plus que sur celle-ci et qui sont 'tous' au surplus 'des esclaves d'un seul homme,' le roi. ${ }^{45}$ En suivant les lois de la nature, le bon Sauvage trouve en lui une morale qui lui permet à la fois de faire l'économie d'une religion ${ }^{46}$ et de dénoncer, au nom 
d'une exigence d'égalité, l'autorité absolue de la monarchie de droit divin. ${ }^{47}$ Absence de propriété, universalité de la raison, inutilité de la religion, rejet de l'autorité monarchique, enfin revendication de liberté et d'égalité définissent en fait les contours du portrait d'un Adario fort peu Sauvage..$^{48}$ Une fois le modèle posé, on le retrouve partout, parfois pous mettre l'accent sur la fonction critique du personnage ou sur son rôle revendicateur. Mais alors la figure est pure fiction, d'ailleurs souvent intégrée à des aventures elles-mêmes fictives, comme l'illustrent celle de lord Tonkson dans les Voyages et aventures $d u$ Chevalier de $* * *$ : obligé de séjourner chez les Caraibes, le héros du récit remarque:

insensiblement je m'accoutumai avec eux, \& ils se plurent avec moi; la beauté \& la fertilité du climat, où sans or ni argent, meurtres ni rapines, on peut se procurer aisément tout ce qui est nécessaire, la façon de vivre des habitans, la douceur de leurs moeurs, l'égalité qui regne entre eux, cette liberté enfin, le plus riche des présens de la nature, si chéri de ceux qui en savent bien connoître tout le prix, eurent pour moi des charmes si puissans que je formai dans l'instant la ferme résolution de ne jamais quitter d'aussi généreux hôtes qui, quoiqu'on en dise, sont moins sauvages, plus humains \& plus droits que ces prétendus hommes polis qui sont répandus dans le reste du monde connu. ${ }^{49}$

Certes, il s'agit d'un roman, mais l'histoire' de lord Tonkson témoigne bien de l'inversion qui caractérise l'image du bon Sauvage par rapport à l'homme policé en plus d'en illustrer tout le caractère fictif, sinon utopique. Le bon Sauvage n'est rien d'autre qu'une image inversée de l'homme policé que tous ses traits, modifiables en fonction des nécessités pédagogiques, nient et mettent en doute..$^{50}$

$\mathrm{Au}$ total, c'est par sa différence, construite artificiellement, que l'Amérindien permet une analyse des problèmes de la société européenne. ${ }^{51}$ Aussi définit-il essentiellement un lieu polémique. De fait, chez La Hontan, les 'dialogues' sont engagés, au sens moderne du terme, en ce qu'ils n'appellent pas seulement un peu plus de liberté religieuse, un peu moins de despotisme politique et un peu plus de justice sociale, mais qu'ils revendiquent le droit de tout soumettre à la critique, c'est-à-dire en pratique, au contrôle de la raison. Or en appeler de la raison, c'est rejeter d'emblée les fondements mêmes de la philosophie, de la morale et de la société louisquatorzième ancrés sur la notion d'autorité, celle de la Tradition et celle d'une monarchie de droit divin. Sur ce plan, la contestation paraît absolue et le caractère polémique du personnage du 'philosophe rustique' poussé à sa limite. D'autres voyageurs ne se priveront pas d'exploiter la veine. De là nombre de 'bons Sauvages' qui illustrent l'aliénation sociale, soit en la dénonçant par l'exemple de leur propre disgrâce, soit en l'analysant 
à la façon d'Adario. ${ }^{52}$ Dans le premier cas, on trouve l"Ancien' des Natchez de l'Histoire de la Louisiane de Le Page du Pratz dont la harangue pathétique, à la veille de la dernière grande révolte amérindienne sous le régime français, traduit le désarroi et la sénescence d'une nation réduite à la vassalité par des maitres qui la parquent dans une réserve et qui lui imposent des valeurs et des modes de pensée qui la détruisent..$^{53}$ Il est vrai qu'ici, c'est moins un philosophe qui parle qu'un homme déjà déchu, mais conscient de son aliénation et qui dénonce à travers ses propres maux le malheur de la civilisation. Chez les voyageurs, en contact plus ou moins étroit avec de véritables Amérindiens, l'image du Sauvage se ressent de la situation réelle dans laquelle vivent les tribus et le plus souvent, la critique demeure ponctuelle. Et même quand l'Amérindien prend la parole en tant que philosophe, c'est surtout pour dénoncer des institutions, des pratiques et des préjugés précis. Tel est le cas du cacique Alibamon qui discute de guerre et de stratégie diplomatique avec le narrateur des Nouveaux Voyages aux Indes occidentales (1768): par amitié pour les Français, le cacique prétend monter une expédition punitive contre les Espagnols de Pensacola parce qu'ils commercent avec les Anglais, alors en guerre contre la France. ${ }^{54}$ Or le narrateur demande à son interlocuteur d'abandonner son projet puisque l'Espagne et la France ne sont pas en guerre. Mais toute la scène ne sert en fait que d'introduction à une discussion qui pose le problème de la justice en fonction de la raison d'État. ${ }^{55} \mathrm{Il}$ est remarquable cependant que le caractère polémique s'atténue sensiblement par rapport à l'oeuvre de La Hontan car dans ce dialogue, à toutes fins utiles, Jean Bossu renvoie dos à dos les deux façons de penser la justice et la raison d'État sans vraiment trancher.

L'image du bon Sauvage se nuance, surtout qu'elle n'était originellement idéalisée sur le plan intellectuel que pour mieux contredire les thèses des missionnaires. En fait, au cours du siècle, un certain relativisme transparait qui recentre en quelque sorte le portrait de l'Autre. C'est que l'image du Sauvage philosophe résiste mal à l'épreuve des faits qui appellent une relecture et une nouvelle analyse. En pratique, tout en continuant à user du bon Sauvage comme instrument d'ordre rhétorique en vue de dénoncer les méfaits de la civilisation ou les conséquences de la superstition et de l'injustice, les relations de voyages, surtout dans la seconde moitié du siècle, produisent des données moins en vue d'une polémique qu'en vue d'une réflexion sur le monde sauvage. En ce domaine, il est vrai autant par opposition aux philosophes que par attachement à la tradition des Relations qui font presque du Sauvage une bête à humaniser puis à convertir, le Journal de voyage de François-Xavier de Charlevoix joue un rôle non négligeable dans l'évolution des idées à l'endroit des 'Américains.' Pour la 
première fois, quoique après l'ouvrage apologétique du père Lafitau dont il s'inspire fortement, un écrivain tente de produire une synthèse qui fasse la part des choses et qui définisse mieux les mours et les pratiques sociales des Amérindiens en fonction de leur mode de vie et des circonstances de leur histoire. De là l'étonnant 'Portrait en racourci des Sauvages' qui accumule toute une série d'oppositions pour rendre compte de leur caractère:

avec un extérieur sauvage, des manieres \& des usages, qui se sentent tout-àfait de la barbarie; on remarque en eux une société exempte de presque tous les défauts, qui alterent si souvent la douceur de la nôtre. Ils paroissent sans passion, mais ils font de sang-froid, \& quelquefois par principe, ce que la passion la plus violente \& la plus effrenée peut inspirer à ceux, qui n'écoutent plus la raison. Ils semblent mener la vie du monde la plus misérable, \& ils étoient peut-être les seuls heureux sur la Terre, avant que la connoissance des objets, qui nous remuent \& nous séduisent, eût réveillé en eux une cupidité, que l'ignorance retenoit dans l'assoupissement, \& qui n'a pourtant pas encore fait de grands ravages parmi eux. On apperçoit en eux un mélange des mours les plus féroces \& les plus douces, des défauts de Bêtes carnacieres, \& des vertus $\&$ des qualités de coeur \& d'esprit, qui font le plus d'honneur à l'Humanité. On croiroit d'abord qu'ils n'ont aucune forme de gouvernement, qu'ils ne connoissent ni loix, ni subordination, \& que vivant dans une indépendance entiere, ils se laissent uniquement conduire au hasard \& au caprice le plus indompté; cependant ils jouissent de presque tous les avantages, qu'une authorité bien réglée peut procurer aux Nations les plus policées. Nés libres \& indépendans, ils ont en horreur jusqu'à l'ombre du pouvoir despotique, mais ils s'écartent rarement de certains principes \& de certains usages, fondés sur le bon sens, qui leur tiennent lieu de Loix, \& qui suppléent en quelque façon à l'autorité légitime. Toute contrainte les révolte, mais la raison toute seule les retient dans une espèce de subordination, qui pour être volontaire, n'en atteint pas moins au but, qu'ils se sont proposé. ${ }^{56}$

Ce 'portrait' manifeste autant la dichotomie des moeurs des Amérindiens, en partie aliénés au contact de la civilisation blanche, que la difficulté des Européens à comprendre la différence et la singularité de l'Autre, voué à l'inintelligibilité absolue. Dans le Journal de Charlevoix, l'Autre demeure d'une certaine façon une énigme, mais une énigme dont les contours, malgré tout, sont si bien circonscrits qu'il est impossible de la faire parler ou du moins de lui faire signifier exclusivement la raison. Dès lors, le 'Sauvage' ne peut plus être le 'bon Sauvage,' porteur d'une rationalité philosophique. Le souci d'exhaustivité qui anime l'historien de la Nouvelle France l'amène à signaler les ombres du tableau qui rendaient possible l'ébauche de la figure 
mythificatrice du 'bon Sauvage,' sinon même à les éclairer par de longs développements sur les moeurs: en somme, la nomenclature interdit le rêve. ${ }^{57} \mathrm{Au}$ surplus, après 1745 , le témoignage et l'autorité d'un La Condamine, de retour d'Amérique du Sud, rendent impossible la persistance de la figure du Sauvage philosophe, d'autant plus que les Amérindiens, définitivement aliénés par l'avancée coloniale européenne, ne présentent plus que des lambeaux de leur antique grandeur. ${ }^{58}$ Du reste, et c'est un constat que font les voyageurs, surtout ceux qui parcourent les 'Isles' et l'Amérique du Sud, les Amérindiens disparaissent: abrutis par l'éthylisme, décimés par les maladies, chassés par les colons, il s'évanouissent littéralement dans la nature, là où les Blancs ne les peuvent pratiquer. C'est donc en grande partie au nom de la science que les voyages mettent un terme à l'image, sinon à l'espoir du 'bon Sauvage' et qu'ils inversent le rapport entre l'Amérindien et le Blanc, redevenu porteur de valeurs positives. Non seulement l'observation et l'analyse nuancent-elles l'image édulcorée produite par les philosophes de cabinet, mais en plus la disparition des mythes est inscrite dans l'activité scientifique: elle fait reculer les frontières des 'terra incognita,' propices aux rêves, ${ }^{59}$ et surtout elle s'allie de plus en plus à un mode de pensée qui prône de nouvelles valeurs et qui modifie par là la perception que l'on se fait de l'Autre. Si chez La Hontan la propriété est à l'origine des maux de l'humanité, chez les 'idéologues,' dont les théories marquent tout le dernier tiers du siècle, c'est au contraire la propriété qui civilise. Alors qu'il dresse un portrait pour le moins sévère des Tlingits aperçus au Port des Français en Alaska, Lapérouse ajoute:

Les philosophes, auront beau se recrier contre ce tableau; ils font leurs livres au coin de leur feu, et je voyage depuis trente ans. Je suis temoin des injustices, de la fourberie de ces peuples qu'on nous peint si bons, parcequ'ils sont trèz prèz de la Nature; mais cette Nature n'est sublime que dans ses masses, elle neglige tous les details; il est impossible de pénétrer dans les bois que la main des hommes civilisés n'a point élagués: de traverser les plaines, qui quoique couvertes de verdures, sont remplies de pierres, de rochers, et inondées de marais impracticables; de faire société enfin, avec les hommes qui sortent de ces mains, parcequ'ils sont barbares, mechants, et fourbes; et qu'il m'est permis d'avoir cette opinion, puisqu'elle ne m'a pas servi à croire, que je pouvois leur apprendre au depends de leur propre vie, qu'il étoit un droit des gens, pour nous comme pour eux. ${ }^{60}$

La condamnation ne repose pas seulement sur l'expérience acquise; elle se fonde surtout sur une ontologie qui va précisément à l'encontre des thèse pastorales: 
Ces Indiens ont fait de beaucoup plus grands progrès dans les arts que dans la morale, et leur industrie, est plus avancée, que celle des habitants des isles de la Mer du Sud, à l'exception cependant de l'agriculture, qui en rendant l'homme casanier, assurant sa subsistance, et lui laissant la crainte, de voir ravager la terre qu'il a plantée, est peut-être plus propre qu'aucun autre moyen à adoucir les moeurs, et à le rendre sociable. ${ }^{61}$

À une conception sociale inverse de celle que formule et fixe en somme le Discours sur l'origine de l'inégalité, répond une vision inversée de l'Autre.

Mieux, assuré de la supériorité du monde policé sur le monde sauvage, on prétend civiliser l'Autre en lui imposant les propres valeurs de l'Europe:

mais seroit-il impossible à un zèle ardent, et à une extrême patience, de faire connoitre les avantages d'une société fondée sur le droit des gens, à un petit nombre de familles? d'établir parmi elles un droit de propriété si seduisant pour tous les hommes, ce qui engageroit chacqu'un à cultiver avec émulation son champ, ou à se livrer à tout autre genre d'industrie? ${ }^{62}$

Au total, l'Autre n'a rien de mieux à souhaiter que de se soumettre aux instructions de l'homme policé et c'est même essentiellement en tant qu'objet d'une mission civilisatrice qu'il intéresse et non plus en tant que témoin d'un ailleurs:

la conquête du nouveau continent (écrit le prince de Nassau-Siegen), l'enfance dans la qu'elle on a trouvé l'amerique relativement aux sciences et aux arts, la variété des races d'hommes qui habitent ces Vaste regions, l'Education que des Législateurs rois et prêtres tout à la fois ont donné à une nation américaine, la Vie Sauvage en outre vraiment dans l'Etat de Nature, la régie de ces nations et des colons par les différents ministères sont les objets qui me frapèrent le plus et que je cherchay à connoître. ${ }^{63}$

Si pour La Hontan, l'Europe avait beaucoup à apprendre du monde sauvage, pour les voyageurs de la fin du siècle, c'est l'homme policé qui, imbu de la supériorité que lui confèrent la science, l'ordre politique et le pouvoir économique, doit transmettre les lumières de la raison à l'homme primitif et lui apporter les bienfaits de la civilisation. Un peu à la façon des missionnaires du XVIIe siècle qui allaient annoncer l'Evangile au péril de leur vie, l'explorateur scientifique part à la conquête des esprits et il entend à son tour humaniser le Barbare en lui inculquant les rudiments de sa religion scientiste. À la veille de la Révolution, un nouvel apostolat se concrétise et d'étranges mission- 
naires partent en mer, armés d'instruments scientifiques et techniques, les cales de leurs navires remplies de graines et d'animaux domestiques à distribuer aux pauvres du monde. ${ }^{64}$ Version laïque du 'compelle intrare, ' l'expédition scientifique fonde les prétentions hégémoniques de l'Europe sur des intentions humanistes et avec la naïve brutalité qui le caractérise parfois, le prince de Nassau-Siegen exprime bien ce point de vue d'une Europe qui s'octroie le droit de posséder le monde par la vertu de sa supériorité technologique et scientifique:

la nature a favorisé certaines parties de la terre plus particulierement que d'autres [écrit-il], voila ou il convient actuellement de former de nouveaux Etablissements: ce sera un nouveau Commerce ouvert à la France. Toutes les richesses du globe appartiennent à l'Europe que les Sciences ont rendue souveraine des autres parties: allons donc recueillir cette moisson. ${ }^{65}$

Selon le droit international qui fonde la possession de territoires non plus tant sur la primauté de la découverte que sur leur occupation effective en vue de les faire fructifier, ${ }^{66} l^{\prime}$ Europe transforme le droit d'occuper la terre en devoir, sinon en mission: le sol n'appartient qu'à celui qui sait l'enrichir. La science, plus que la force physique, donne le droit de prendre possession des îles et des territoires 'découverts' et ce d'autant plue que cette même socience fournit les moyens de l'imposer. ${ }^{67}$ C'est alors que l'Amérindien commence à cesser d'être un personnage intéressant et une figure opératoire sur le plan littéraire pour devenir un véritable objet d'étude ethonologique enfin perçu dans sa réalité. Ce que l'image du Sauvage perd en intérêt sur le plan philosophique, elle le regagne dans le champ scientifique et la figure de l'Autre cède d'autant plus facilement sa place au sein des débats polémiques que de nouvelles questions appellent de nouvelles figures, celle du Noir et plus encore celle de l'Insurgent.'

Enfin des facteurs intrinsèques à la réflexion philosophique expliquent la disparition de la figure du Sauvage philosophe. L'Amérindien, qu'il se nomme Adario ou Ingénu, sert essentiellement à l'intérieur d'une polémique fondée sur l'espérance de transformer le monde pour rendre les hommes meilleurs et plus heureux par le biais de la raison. ${ }^{68}$ Or cette foi s'atténue singulièrement après 1750 et plus précisément avec les événements de la guerre de sept ans. Ainsi, dès 1761, l'Histoire d'un bon Bramin illustre le malheur de l'homme qui vit dans le temps sans savoir ce qu'est le temps, qui pense sans comprendre ce qu'est la pensée, qui veut croire le monde perfectible, mais qui n'aperçoit que maladies, misère et mort, enfin qui découvre que la pensée n'est rien d'autre qu'un cadeau empoisonné car si elle aide à poser lucidement les problèmes, elle ne permet jamais de résoudre quelque 
question que ce soit, ni de résorber le mal. Or la figure du Sauvage est précisément liée à l'espoir de transformer le monde par la raison. Aussi quand cette foi s'amenuise, la figure de l'Autre s'estompe. Au demeurant, laminé par l'usage, le 'bon Sauvage' n'est plus qu'un cliché, presque une figure de rhétorique, usée, éculée et qui perd toute valeur. Pis, elle s'inverse et un Cornelius de Pauw peut en 1771 sonner définitivement le glas du 'bon Sauvage' et présenter, au nom d'une conception pré-physiocratique, ${ }^{69}$ une image de l'Amérindien qui en fasse l'exemple même de toutes les disgrâces, pour tout dire un être absolument dégénéré et monstrueux. ${ }^{70}$ Les ripostes de dom Pernety et de Pierre Poivre pour défendre les Amérindiens n'y feront rien: la figure du 'bon Sauvage' est définitivement enterrée. ${ }^{71}$

Paradoxalement, c'est précisément l'amenuisement de la figure qui permet la métamorphose de l'Autre en concept ou plus précisément la résurgence du concept et la relance de la problématique sur de nouvelles voies dans la seconde moitié du siècle. À force de s'apetisser comme image, l'Autre devient une abstraction, à force de disparaître de la réalité, il s'estompe comme s'il appartenait non seulement à un autre espace, mais aussi à un autre temps. Selon un monde de pensée issue de la pratique des textes bibliques dans lequel la distance géographie correspond réellement à un éloignement chronologique, ${ }^{72}$ les penseurs au XVIIIe siècle établissent une relation entre la distance géographique et la distance chronologique: pour eux, l'éloignement des Amérindiens par rapport au continent européen marque en fait une distance chronologique qu'ils traduisent par la notion de primitivité et d'enfance, et ce d'autant plus facilement que les analyses d'ordre psychologique d'alors reconnaissent les phases d'évolution de chaque individu dans celles de l'espèce et qu'elles retrouvent alors dans l'homme primitif les signes de l'enfance de l'humanité. C'est ainsi que le Sauvage se métamorphose en témoin vivant du passé perdu de $\mathrm{l}^{\prime}$ Homme. ${ }^{73}$ Dans cette perspective, l'Autre représente une sorte d'alpha, ou si l'on préfère, le point de départ des sociétés appelées à se policer et il se retrouve au magasin des idées. Alors l'Amérindien devient 'une image parfaite des premiers tems ... de la naissance du monde. ${ }^{74} \mathrm{~L}$ 'Autre se transforme en concept et il vient à constituer une abstraction échappée au temps, à l'espace et à la corruption. Les penseurs, beaucoup plus que les voyageurs du reste, n'auront qu'à s'emparer de l'idée qu'il est devenu pour poser en termes philosophiques le problème de l'évolution de l'homme. Ici toutefois, l'Autre est moins le Sauvage que le Primitif, que les circonstances associent d'ailleurs plutôt aux insulaires du Pacifique et de plus, c'est toujours sous forme de palimpseste en quelque sorte qu'il apparaît dans la mesure où ceux qui créent et qui pensent le concept effacent, ou du moins oublient 
le discours des voyageurs, pour inscrire de nouveaux signes sur le texte. ${ }^{75}$

Déjà en 1651, Thomas Hobbes entreprenait de définir 'l'état de nature' en vue de fonder en droit l'existence de la société. En effet, puisque ' l'état naturel des hommes, avant qu'ils eussent formé des sociétés, étoit une guerre perpétuelle, \& non seulement cela, mais une guerre de tous contre tous, ${ }^{76}$ il devenait indispensable de former des sociétés pour éviter l'anéantissement de l'espèce. Cependant, avec Thomas Hobbes, l'homme de la nature n'est pas, peu s'en faut, une pure abstraction. L'exemple des 'Américains' témoigne hors de tout doute du caractère bien concret de l'homme de la nature dans la pensée de Hobbes. ${ }^{77}$ Mais si l'homme de la nature ressemble à s'y méprendre au Sauvage sans lois et sans organisation sociale, tel que le suppose Hobbes, en revanche, les lois de nature paraissent parfaitement abstraites. Les vingt lois de nature que définit le $\mathrm{De}$ Cive et qui servent en réalité de prémisses morales nécessaires à l'établissement de toute société 78 'ne sont pas des loix à parler proprement, en tant qu'elles procèdent de la nature \& considérées en leur origine. Car elles ne sont autre chose que certaines conclusions tirées par raisonnement touchant à ce que nous avons à faire ou à omettre. ${ }^{\prime 79} \mathrm{Si}$ conceptualisation il y a chez Hobbes, ce n'est pas au niveau de la définition de l'homme primitif qu'elle s'effectue, mais dans l'élaboration des postulats éthiques jugés indispensables au maintien de la cohésion sociale. Mieux, les lois de nature n'ont de valeur, paradoxalement, qu'autant qu'elles permettent aux hommes de sortir de l'état de nature. ${ }^{80} \mathrm{Il}$ n'empêche. L'homme de la nature est inscrit dans une réflexion et il prend déjà valeur de théorème au sein d'une méditation philosophique et rationaliste. Il suffira de la dépouiller de ses attributs encore concrets pour le transformer en pur concept, au même titre que la 'loi morale' obtenue 'par calcul rationnel, par ratiocination. ${ }^{81}$ John Locke se chargera en 1691 de l'opération. En effet, dans son traité $D u$ Gouvernement civil, Locke a recours à l'idée d'homme primitif pour démontrer que l'état de nature constitue un état idéal dans lequel le droit individuel, antérieur à toute forme de socialisation, prime sur l'ordre social et que le pacte qui institue la société a précisément pour objet de garantir les droits naturels, notamment celui, imprescriptible, de propriété. ${ }^{82}$ Tout comme Hobbes, avant lui, et Rousseau, après lui, John Locke formule le concept d'homme primitif pour des raisons d'ordre épistémologique. Il cherche à définir les attributs fondamentaux de l'homme à partir de l'état de civilisation qu'il connaît en en retranchant les ajouts artificiels qu'il lui suppose. En toute logique, puisque l'état policé est le résultat d'additions à l'état originel et le produit de l'arbitraire, en gommant ceux-ci, on doit pouvoir esquisser l'état de nature. Ainsi le concept d'homme primitif n'est rien d'autre 
qu'une invention ordonnée à partir de l'état de culture à laquelle on retire des éléments et dont on élague ce qu'on croit lui avoir été ajouté au cours des âges. Si en principe, l'état de culture provient de l'état sauvage, dans la démarche intellectuelle du philosophe, c'est l'inverse qui se produit quand il est question de définir l'homme primitif: c'est à partir de la réalité qu'on a sous les yeux et qu'il s'agit de légitimer (Hobbes), de critiquer (Locke) ou de dénoncer (Rousseau) qu'on définit les attributs de l'homme primitif. À la vérité, tout le concept d'homme primitif est inclus dans la réalité qui sert de point de départ à l'analyse. L'Autre n'est rien de plus qu'une tension issue d'une dialectique entre un idéal à instaurer et la réalité contemporaine. Il est ce 'modèle absent,' pour reprendre un terme de François Hartog au sujet de la représentation de l'Autre chez Hérodote, ${ }^{83}$ qui sert de référence $a b-$ straite en vue de comprendre la réalité et de construire un discours sur elle. L'Autre est le résultat d'un bricolage intellectuel réalisé à partir du connu. C'est bel et bien ce qui se passe chez Locke chez qui l'analyse s'inscrit dans un débat jusnaturaliste qui entend poser non seulement les conditions d'existence de la société, mais en même temps les conditions de toute réflexion sociale. Chez lui, le discours ne porte pas seulement sur le procès de fondation de la société, mais tout autant sur le procès de fondation de l'analyse. On conçoit à quel niveau d'abstraction mène ce mode de pensée. ${ }^{84}$ Ainsi l'état de nature est-il essentiellement défini par rapport à l'état de société comme un manque, si bien que l'état de nature existe non seulement pour John Locke dans les sociétés primitives, mais qu'il existe partout où un corps politique n'est pas dûment constitué: 'toutes autres sortes d'engagements \& de traitez, que les hommes peuvent faire entre eux, les laissent dans l'estat de nature, ${ }^{\prime}{ }^{85}$ observe Locke qui produit des exemples qui n'ont guère à voir avec les Amérindiens ou les primitifs puisqu'à ses yeux, en l'absence de contrat social passé entre les parties, une monarchie absolue ne saurait constituer un état politique. Entre les Sauvages qui troquent entre eux des produits en réglant leurs exigences sur les lois instinctives de la justice ${ }^{86}$ et des Européens qui subissent le joug d'une monarchie absolue, il n'y a aucune différence d'essence: 'là seulement se trouve une Société politique où chacun des membres s'est dépouillé de son pouvoir naturel, \& l'a remis entre les mains de la Société, afin qu'elle en dispose dans toutes sortes de causes qui n'empeschent point d'appeler toûjours aux loix établies par elle. ${ }^{187} \mathrm{Au}$ total, ceux qui peuvent en appeler à des lois, parce qu'elles fondent le pouvoir et qu'elles sont au-dessus des individus dans lesquels se réalise le pouvoir, vivent dans l'état de société, 'mais ceux qui ne peuvent appeler de mesme à aucun tribunal sur la terre, ni à aucunes loix positives, sont toûjours dans l'estat de nature. ${ }^{\prime 88}$ Pour résumer, 'les hommes donc sortent de 
l'estat de nature, \& entrent dans une Société politique, lors qu'ils créent \& établissent des Juges \& des Souverains sur la terre, à qui ils communiquent l'autorité de terminer tous les differens, \& de punir toutes les injures qui peuvent estre faites à quelqu'un des membres de la Société. ${ }^{89}$ Sans entrer ici dans le détail des intentions de John Locke qui entend limiter le pouvoir monarchique anglais au nom du principe du contrat social, il est intéressant de remarquer que le concept d'état de nature dépasse de beaucoup l'idée de peuple primitif puisqu'il englobe en fait des états fort peu primitifs au sens originel du terme. En fait l'état de nature est partout où il n'y a pas de contrat social passé entre les parties et pour John Locke, qui prétend fonder en droit la monarchie constitutionnelle anglaise, l'état de nature ne peut être autre chose qu'un 'estat de parfaite liberté,' un 'estat aussi d'égalité' 90 qui exclut au départ toute subordination car c'est seulement à la condition d'avoir pour contractants des hommes libres et égaux que peut se fonder le droit de la monarchie constitutionnelle, celui de la propriété et de la liberté auxquels croit le philosophe.

On comprend que Jean-Jacques Rousseau soit amené à inventer le concept d'état de pure nature, antérieur à toute socialité, pour ancrer ses théories sur des principes qui ne soient pas tirés de l'état de société. En effet, John Locke ne part pas du passé pour en déduire des principes applicables au présent; c'est la démarche inverse qu'il accomplit. Il invente un concept d'état de nature et il investit l'homme primitif d'attributs précis pour fonder en droit des principes auxquels il croit avant même d'aborder la question. Inscrite à l'intérieur d'un essai philosophique et non polémique, la figure de l'Autre subit l'influence du mode de pensée de l'ouvrage et elle vire au concept abstrait qui permet de postuler l'Homme comme fin de toute société et de tout gouvernement. Chez Locke, l'Autre se transforme en proposition syllogistique en vue de définir une conception de l'État, et il formule un archétype à usage méthodologique. ${ }^{91}$ En France, l'idée est reprise et si Denis Diderot, par ses positions sensualistes et matérialistes, maintient un semblant de présence à son 'Otaïtien' pour démontrer le rôle que jouent les lois dans l'orientation du comportement moral des hommes ${ }^{92}$ et pour dénoncer la problématique tragique de la socialisation de l'humanité qui s'est faite à l'encontre de la nature originelle de $l^{\prime}$ homme, ${ }^{93}$ chez la plupart des autres penseurs, notamment chez Morelly ${ }^{94}$ et surtout chez Jean-Jacques Rousseau, l'homme primitif luimême tend à la pure abstraction, pour tout dire à la catégorie ontologique. ${ }^{95}$ Comme l'écrit explicitement Rousseau, l'état de pure nature renvoie à 'un état qui n'existe plus, qui n'a peut-être point existé, qui probablement n'existera jamais, et dont il est pourtant nécessaire d'avoir des notions justes, pour bien juger de notre état présent. ${ }^{96}$ Tout ce 
que Jean-Jacques Rousseau affirme de l'état originel de l'homme provient d'une réflexion abstraite, d'une méditation en vue de retracer les principes antérieurs à la raison même. ${ }^{97}$ En vérité ce mode d'approche paraît nécessaire à qui veut fonder un discours original sur l'homme: c'est pour dépasser les thèses de Thomas Hobbes, qui conçoit l'état de nature comme un état de guerre, celles de John Locke, de Buffon, de Diderot, enfin d'Helvetius qui tous conçoivent l'homme primitif proche de l'état d'animalité et vivant déjà en groupes que Jean-Jacques Rousseau invente un état antérieur à cet 'état de troupeau'; l'état de pure nature, alors que les hommes vivent solitaires et disséminés sur la surface de la terre. Comme le rappelle Michèle Duchet, Buffon, Diderot et Helvétius 'admettent tous trois que les premiers hommes ont vécu dans un état proche de l'animalité, tandis que pour Rousseau l'animal humain se distingue d'abord de tous les autres parce qu'il ne vit point naturellement en troupe. ${ }^{\prime 98}$ De là la nécessité heuristique d'une abstraction supplémentaire afin d'inventer un état antérieur, en fait ontologique, de l'homme: l'état de pure nature est indispensable à Rousseau pour décrire et pour fonder les caractères fondamentaux, essentiels de l'être humain. Et pour réaliser sa démonstration, Rousseau procède par abstractions successives:

Commençons donc par écarter tous les faits; [écrit-il en tête de son analyse] car ils ne touchent point à la question. Il ne faut pas prendre les recherches, dans lesquelles on peut entrer sur ce sujet, pour des vérités historiques, mais seulement pour des raisonnements hypothétiques et conditionnels, plus propres à éclaircir la nature des choses qu'à en montrer la véritable origine, et semblables à ceux que font tous les jours nos physiciens sur la formation du monde..$^{99}$

Rousseau fonde un modèle épistémologique propre à son analyse anthropologique comme d'autres, en sciences, créent des modèles mathématiques pour rendre compte de phénomènes physiques. Michèle Duchet:

On s'est souvent demandé si "l'homme sauvage" de Rousseau était une pure abstraction, ou l'homme des premiers temps. Il nous semble qu'on a trop insisté sur le caractère "hypothétique" de l'état de nature, selon Rousseau, en oubliant la logique qui fonde ce recours à l'hypothèse. La démarche du Second Discours est en effet tout à fait comparable à celle d'un dialogue platonicien: s'éloignant des "faits" par une lente ascension, pour se former une idée de l'homme sauvage qui soit véritablement "propre à éclaircir la Nature des choses. ${ }^{\prime \prime 100}$ 
C'est ainsi que l'état de pure nature n'est rien d'autre qu'une hypothèse de travail, ce que Henri Gouhier signalait déjà dans les Méditations métaphysiques de Jean-Jacques Rousseau: 'Si l'état de nature n'est pas une époque historique mais une hypothèse de travail, $c^{\prime}$ est une hypothèse de travail faite pour comprendre l'homme historique; si l'état de nature ne représente pas "la véritable origine," il se présente comme une origine hypothétique. ${ }^{\prime 101}$ Résultat d'un élagage, issu d'une opération mentale peut-être d'ailleurs inspirée d'une remarque de Hobbes, ${ }^{102}$ absolument abstrait dans son principe, l'état de pure nature n'a donc rien à voir avec la réalité sauvage, si primitive paraisse-t-elle parfois: en effet, les 'peuples sauvages' sont déjà loin du premier état de nature,' constate Rousseau. ${ }^{103}$

Et pourtant. Comment expliquer l'engouement des uns et des autres à l'endroit de cet état abstrait hissé au rang de mythe, voire devenu une aspiration? Comment le modèle heuristique est-il devenu si facilement une robinsonnade? Certes

l'expérience capitale de l'Essai, c'est donc celle d'une socialité heureuse, d'une communication à la fois tendre et vive, d'un langage total, où le geste, la danse et les accents passionnés expriment le désir et le plaisir "confondus," sans distance ni absence, auprès du cristal des fontaines, ou du feu sacré, dans la pureté et la transparence. [Certes] c'est là que Rousseau rencontre, pour la première fois, dans la clarté de l'imaginaire, ce paysage champêtre et patriarcal, tout vibrant de "signes vocaux," et ces rites du foyer et de la danse, que la vie à Clarens ordonnera dans une même symphonie. ${ }^{104}$

Mais tout ceci n'explique pas vraiment le passage de l'epistêmê au rêve. Mais peut-être le paradigme lui-même n'est-il pas sans ambiguiité chez Rousseau. Bien sûr l'état de pure nature est une vue de l'esprit, mais il n'en comporte pas moins des traits nets sur lesquels pouvaient se greffer tous les rêves des hommes en proie au désir d'un monde heureux et bon, d'un monde composé d'une humanité enfin réconciliée avec elle-même: tout abstrait que paraisse l'homme de la pure nature, Jean-Jacques Rousseau n'est pas sans l'apercevoir distinctement: 'je vois un animal ... Je le vois. ${ }^{105} \mathrm{C}^{\prime}$ est que le modèle n'est pas si abstrait qu'il paraît au départ et que pour le formuler, Rousseau n'a pas fait qu'élaguer les faits et raisonner; il a aussi abondamment puisé dans les relations des voyageurs et dans l'Histoire générale des voyages et il a souvent appliqué à son Homo solivagus des caractéristiques empruntées à l'Homo sylvestris. ${ }^{106}$ Pas tout à fait idéel cet homme dans l'état de pure nature qui ressemble parfois, à s'y méprendre, aux Sauvages dont Rousseau évoque les traits. Notamment les Caraibes, 'celui de tous les peuples existants qui jusqu'ici s'est écarté le moins de l'état 
de nature' et que Rousseau présente paisible dans ses amours et doux de caractère. ${ }^{107}$ Le rapport est si étroit entre les deux états de nature et de sauvagerie que Rousseau lui-même verse dans le rêve en évoquant l'époque heureuse d'une humanité sauvage, sortie à peine de l'état de pure nature, mais pas encore engagée dans l'histoire avec ses maux et ses inégalités, figée dans l'intemporalité lumineuse du bonheur:

Ainsi, quoique les hommes fussent devenus moins endurants, et que la piété naturelle eût déjà souffert quelque altération, cette période du développement des facultés humaines, tenant un juste milieu entre l'indolence de l'état primitif et la pétulante activité de notre amour-propre, dut être l'époque la plus heureuse et la plus durable. Plus on y réfléchit, plus on trouve que cet état était le moins sujet aux révolutions, le meilleur à l'homme, et qu'il n'en a dû sortir que par quelque funeste hasard, qui, pour l'utilité commune, eût dû ne jamais arriver. L'exemple des sauvages, qu'on a presque tous trouvés à ce point, semble confirmer que le genre humain était fait pour y rester toujours, que cet état est la véritable jeunesse du monde; et que tous les progrès ultérieurs ont été, en apparence, autant de pas vers la perfection de l'individu, et, en effet, vers la décrépitude de l'espèce. ${ }^{108}$

La robinsonnade n'est possible que parce que l'état de pure nature emprunte à l'état sauvage et qu'il existe, du moins dans l'imagination de Rousseau, un moment privilégié où l'homme, sans avoir perdu toutes ses qualités originelles, mais déjà réuni en microsociétés vaguement paternalistes à travers les échanges d'une aimable civilité, sans rapports de forces, a vécu en dehors de l'histoire un bonheur sans faille. Du modèle abstrait, trop souvent caractérisé par des emprunts à la réalité sauvage telle qu'elle apparaît dans ce qu'elle présente de plus fortuné, Rousseau établit lui-même la jonction avec le mythe d'une sauvagerie heureuse, ou plutôt avec le mythe d'une nature radieuse, et il renoue avec un idéal qui paraît soudain à portée de la main. Finalement, comme le monde sauvage semble faire l'économie de l'écriture et de pacte social arrêté, les ajouts propres au monde policé, il répond assez à l'idée de l'état de pure nature, même si en fait les cultures amérindiennes ou océaniennes reposent sur des règles précises et complexes, même s'il faut se garder 'de confondre l'homme sauvage avec les hommes' de l'état de pure nature. ${ }^{109}$

Ce n'est pas ici le lieu d'exposer les théories défendues par Denis Diderot, par Morelly, par Condillac, par d'Holbach, par Gros de Baplan ou par Jean-Jacques Rousseau, d'autres l'ont fait,, 110 mais il est intéressant d'observer qu'aucun d'entre eux n'a développé ses thèses à l'intérieur d'une relation de voyage. Le fait n'est pas sans importance. Pour 
penser le concept d'homme primitif, il a fallu s'abstraire de l'expérience concrète du voyage, il a fallu voyager à l'intérieur des voyages, ou plus exactement à l'intérieur des relations de voyages. C'est une lecture au second degré que pratiquent les penseurs qui pillent les relations de voyages pour asseoir leurs thèses. Mais ironiquement, leurs théories ne sont pas sans exercer une influence sur la perception que les voyageurs se font de l'Autre. On l'a vu chez Bougainville qui ajuste ses descriptions aux théories rousseauistes, ${ }^{111}$ on peut le voir aussi en suivant l'évolution du rapport que les voyageurs établissent avec le 'Sauvage' ou du moins avec sa figure. ${ }^{112}$ Toutefois, dans les relations de voyages, on fait alors l'économie du concept au profit de l'invention d'un mythe, comme si on traduisait les théories en faits, ou plutôt comme si les concrétisait à travers la résurgence du mythe édenique d'une nature vierge qui a échappé à la tare originelle. Alors qu'il observe des Caraibes, Gautier du Tronchoy note que faute de besoins avant l'arrivée des Européens, ils vivaient sans angoisse, sans inquiétude, sans conscience du mal, en somme qu'ils 'menoient à [s]on avis la vie du monde la plus heureuse. ${ }^{113} \mathrm{Ils}$ évoquent, on s'en doute, l'état de l'homme avant la chute, sinon la possibilité d'une existence en marge du plan divin. ${ }^{114}$ En fait le mythe n'a jamais complètement disparu des esprits. En revanche, ce qui est nouveau, c'est qu'on va moins tenter de résorber le scandale ontologique qu'il formule, comme le font les missionnaires, que de se laisser aller au rêve en développant et en exploitant les conséquences du concept d'homme primitif. À telle enseigne que parallèlement à la figure du Sauvage philosophe, le siècle développe une forme de projection sur le Sauvage de l'espoir d'une humanité heureuse. Ainsi, au prix parfois de singulières contradictions, l'Amérindien se transforme en concrétion d'un désir. ${ }^{115}$ Mieux il devient une valeur refuge qui permet de rêver un monde, non pas seulement meilleur, mais débarrassé du mal, et il permet une image de soi-même délestée de la mauvaise conscience et libérée de la malédiction divine. À tout prendre, il forme 'un espace susceptible d'être habité par tout discours ethnologique fondé sur la nostalgie, la découverte de soi en l'autre et de l'autre en soi, le rêve de l'origine, la certitude d'atteindre, à travers des sociétés de plus en plus "primitives" le passé de l'espèce. ${ }^{\prime 116}$ Plutôt que des Amérindiens, les voyageurs rencontrent des formes vides, sortes de corps habitables par le discours et par le rêve qui le provoque. ${ }^{117}$

On comprend que le mythe se distende en quelque sorte et qu'il prenne de plus en plus la dimension et la forme d'un espace véritable. Cette spatialisation se réalise surtout, il faut le reconnaître, dans le roman, et très tôt, notamment dans les ouvrages de l'abbé Prévost. Pour 
s'en tenir à un seul exemple, au moment où il assure Manon qu'il est prêt à la suivre jusqu'au bout du monde, le chevalier Des Grieux explique:

Je me supposais déjà, avec elle, dans une région inculte et habitée par des sauvages. Je suis bien sûr, disais-je, qu'il ne saurait y en avoir d'aussi cruels que G...M... et mon père. Ils nous laisseront du moins vivre en paix. Si les relations qu'on en fait sont fidèles, ils suivent les lois de la nature. Ils ne connaissent ni les fureurs de l'avarice, qui possèdent G...M..., ni les idées fantastiques de l'honneur, qui m'ont fait un ennemi de mon père. Ils ne troubleront point deux amants qu'ils verront vivre avec autant de simplicité qu'eux. ${ }^{118}$

L'idée sera reprise par d'autres romanciers, notamment à la fin du siècle par Claude Joseph Dorat qui évoque les 'Peuples que nous appelons Sauvages,' mais qui, pour les amants fortunés et poursuivis que sont la marquise de Syrcé et le comte de Mirbelle, se montrent non seulement humains, mais même maternels:

ils nous recevront [croit le comte désespéré], ils nous plaindront, ils admireront notre fermeté, notre amour, notre dévouement courageux aux lois saintes dont l'infraction sacrilège, en vous épargnant le supplice de rougir, nous aurait laissé des remords plus horribles que lui. Aux extrémités de la terre, sous quelque climat que nous habitions, nous trouverons une bonté naturelle qui nous fera grâce, qui soulagera nos maux. Nous trouverons, non des lois établies par des hommes aveugles et barbares, mais la sensibilité vraie, mobile universel de tout être que nos politiques institutions n'ont point dégradé. L'opprobre ne nous suivra point; il restera, chère amante, à ceux qui voulaient vous en accabler. Ma femme, oui, ma femme à mes côtés, mon enfant dans mes bras, je ne serai nulle part étranger. Les lieux où l'on s'attendrit sur l'infortune, voilà notre patrie. ${ }^{119}$

L'homme naturel se confond avec le lieu dans lequel les malheureux peuvent se reposer du tumulte du monde et trouver un havre de paix. Insensiblement, on passe du mythe de l'homme primitif, prêt à recevoir les malheureux, à l'invention d'une nature-refuge. Ainsi dans la relation de voyage de Malouet, est-ce la nature et elle seule qui permet à l'homme de vivre en osmose avec l'univers et avec son créateur, comme si par le détour d'un mythique passé, se réalisait enfin au présent l'Oukoumène future:

C'est à l'occident [écrit Malouet] qu'on retrouve le monde primitif, la terre et les hommes, dans leur état naturel. Là se fait entendre dans la solitude la voix du Créateur, et l'on sent de toute part la puissance de son bras invisible. Là 
vous découvrez la forme native du globe et ses traits originaux, l'union intime de la terre et des eaux, et leur séparation progressive. Ce ne sont point les hommes qui ont chassé l'Océan de cette plage, et qui la couvrent de plantes, d'arbustes et d'arbres divers! Ces dômes de verdure supportés par des colonnes entre lesquelles des lianes se dessinent en festons, cette superbe architecture des forêts est descendue du ciel pour me rendre témoignage de son auteur. ${ }^{120}$

Concrétisation de l'espoir d'un monde pur, le mythe primitiviste se dissout dans le rêve d'une nature accueillante, au sein de laquelle l'homme, enfin réconcilié avec lui-même, fait l'expérience intime de l'eden en effaçant les traces mêmes de l'histoire. ${ }^{121}$

Mais l'Autre, qu'il soit 'Sauvage,' 'bon Sauvage,' pur concept ou mythe, n'a plus rien à voir avec la réalité. Il n'est que littérature.

\section{PIERRE BERTHIAUME}

Université d'Ottawa

\section{Notes}

1 Lapérouse, lettre à Claret de Fleurieu, datée du 7 février 1788, in Maurice de Brossard et John Dunmore, Le Voyage de Lapérouse, 1785-1788 (Paris: Imprimerie nationale, 1985), I, p. 279.

2 Pour Geoffroy Atkinson, la question du 'bon Sauvage' est capitale au XVIIle siècle: 'Le "bon sauvage" appartient, en effet, au XVIIIe siècle dans ce sens que les auteurs de ce siècle lui ont donné son expression littéraire, son expression définitive' (Les Relations de voyages au XVIIe siècle [Genève: Slatkine Reprints, 1972]) p. 63. Voir aussi pp. 63-81.

3 Voir L'Amérique et le rêve exotique dans la littérature française au XVIIe et au XVIIle siècle (Genève: Slatkine Reprints, 1970), pp. 1-220 et 431-33. On aura compris que si la démonstration de Gilbert Chinard est convaincante en ce qui touche le XVIIe siècle, elle nous paraît sujette à caution dans le cas du XVIIIle siècle.

4 Claude Lévi-Strauss, 'Jean-Jacques Rousseau, fondateur des sciences de l'homme,' in Jean-Jacques Rousseau par Samuel Baud-Bovy, Robert Derathé, et al. (Neuchâtel: La Baconnière, 1962), p. 245.

5 'Sauvages et civilisés au XVIIle siècle,' in François Châtelet et Gérard Mairet, Histoire des idéologies (Paris: Hachette, 1978), tomme III, p. 212.

6 Anthropologie et histoire au siècle des Lumières (Paris: Flammarion, 1977), p. 13. C'est l'auteur qui souligne.

7 François Hartog, Le Miroir d'Hérodote (Paris: Gallimard, 1980), p. 249. 
8 Michèle Duchet, op. cit., p. 16. À ce sujet, Jean Ehrard observe que le primitivisme au XVIIle siècle est polémique et allégorique. Voir L'Idée de nature en France à l'aube des lumières (Paris: Flammarion, 1970), pp. 390-95. Ceci dit, on trouve des résurgences de la conception antiquisante du 'Sauvage,' entre autres chez Frézier et chez Bacqueville de la Potherie qui comparent encore les Amérindiens aux Romains et aux peuples de l'Antiquité sacrée. Voir Relation du voyage de la mer du Sud aux côtes du Chily (1716), pp. 73, 109-110 et Histoire de l'Amérique septentrionale (1722), I, pp. 117, 121, 122, 124 et II, pp. 38-9.

9 'Hétérologies' ou 'discours sur l'autre': Michel de Certeau, L'Ecriture de l'histoire (Paris: Gallimard, 1984), p. 10.

10 Op. cit., 1703, I, 31.

11 Ibid., II, 117.

12 On peut dire du discours de l'Autre, lorsque celui-ci est l'étranger par excellence que constitue l'Amérindien, ce que Michel de Certeau écrivait de l'historiographie: 'La seule quête historique du "sens" demeure en effet celle de l'Autre' mais ce projet, contradictoire, vise à 'comprendre' et à cacher avec le 'sens' l'altérité de cet étranger, ou, ce qui revient au même, à calmer les morts qui hantent encore le présent à leur offrir des tombeaux scripturaires': L'Ecriture de l'histoire, p. 8. Ainsi est-ce le silence de l'Autre qui autorise le discours du voyageur. Voir L'Ecriture de l'histoire, pp. 7-13 et surtout son analyse de l'ethnographie' chez Jean de Léry, pp. 215-48.

13 Nouveaux Voyages (1703), II, 91. C'est l'auteur qui souligne.

14 Op. cit. (1770), VI, 44. Plus précisément, en illustrant les mécanismes originels des sociétés, alors que les hommes 'appeloient mystérieux, surnaturel \& céleste, ce qui n'est que l'ouvrage du temps, de l'ignorance, de la foiblesse ou de la fourberie,' les hommes primitifs démontrent la possibilité d'ériger une société en faisant l'économie des idées de propriété et de religion. Voir op. cit., VI, 43-44.

15 Voir Gustave Lanson, 'Origines et premières manifestations de l'esprit philosophique dans la littérature française de 1675 à 1748,' en Revue hebdomadaire des cours et conférences, no. 21 (2 avril 1908), 155, et. no. 23 (16 avril 1908), 245-49.

16 Voir Nouveaux Voyages (1703), I, 249-54. À noter que l'une des originalités de La Hontan consiste à se donner le mauvais rôle, celui du chrétien convaincu, mais maladroit. Le procédé est repris dans les Dialogues dans lesquels le discours de l'auteur, constate Maurice Roelens, 'frôle constamment la parodie' et présente finalement une 'caricature' de la religion. L'idée est 'assez rare et originale,' de conclure le critique. Voir La Hontan, Dialogues avec un Sauvage (Paris: Editions sociales, 1973), introduction, pp. 47-9.

17 'Mais depuis que j'ai raisonné avec ces Peuples,' Nouveaux Voyages (1703), II, 92.

18 Voir Raynal, G.-T.-F., op. cit. (1770), VI, 54. Comme le remarque Maurice Roelens dans son introduction aux Dialogues, on trouve quelques erreurs au sujet de l'âge et de la venue, improbable, d'Adario-Le Rat en France. En outre, le discours du chef amérindien ressemble trop à une 'version "populaire" des critiques et des arguments élaborés dans les milieux intellec- 
tuels de la fin du XVIIe siècle' pour appartenir à un Huron. Au reste, les arguments à coloration protestante (dénonciation des moines et de leur lubricité, ridicule des indulgences, négation de l'autorité papale, rejet du purgatoire, etc.) et les concepts métaphysiques renvoient manifestement davantage au transfuge La Hontan qu'à un Huron. Voir Dialogues (1973), pp. 46-47 et 54-56. D'ailleurs, c'est avec une certaine maladresse que La Hontan justifie le style du discours d'Adario, par trop européen.

19 À noter que l'expression 'philosophes rustiques,' au pluriel, se trouve chez La Hontan. Voir Nouveaux Voyages (1703), II, 104. Au sujet des 'sottises,' voir ibid., II, 99.

20 'Je me suis trouvé cinquante fois avec eux, très-embarrassé à répondre à leurs objections impertinentes' Nouveaux Voyages (1703), II, 113.

21 Sur les rapports entre romans utopiques et la question de la figure du 'bon Sauvage,' voir Gilbert Chinard, op. cit., 188-220. Rappelons que nombre de thèmes exploités par La Hontan - principe du dialogue, axiome de l'existence d'une loi naturelle, distinction entre la nature et les acquis culturels, problème de la propriété - demeurent très proches de ceux développés par Baltazar Gracian dans L'homme détrompé ou le Criticon (Paris: Jacques Collombat, 1696) et par Claude Gilbert dans Histoire de Calejava ou de l'isle des Hommes raisonnables Avec le paralelle de leur Morale $\mathcal{E} d u$ Christianisme (s.I., s.é., 1700). Toutefois il ne faut pas nier l'originalité de La Hontan. Comme le rappelle Michèle Duchet, 'il est le premier à avoir donné forme, dans ses Dialogues, à une contestation du monde civilisé, mais âprement critique, le premier surtout à comparer terme à terme les avantages de l'état sauvage et de l'état de civilisation, dans un dialogue où le personnage du sauvage "raisonneur" tient un rôle essentiel, et non plus seulement épisodique': op. cit., p. 93. C'est l'auteur qui souligne.

22 L'Espion du Grand-Seigneur, et ses relations secretes, envoyées du Divan de Constantinople. Decouvertes à Paris pendant le regne de Louys Le Grand. Traduites de l'Arabe en Italien par le Sieur Jean-Paul Marana de l'Italien en François par *** (Paris: Claude Barbin, 1684). L'origine italienne de l'auteur et son maniement de l'ironie fondent sans doute le sens critique de l'ouvrage. L'idée, remarque Gilbert Chinard, n'est pas neuve puisqu'on la retrouve déjà dans les relations de voyages dès la découverte de l'Amérique. Voir op. cit., p. 179. Toutefois, des influences plus immédiates paraissent marquer La Hontan, notamment celle de Marana. Sur cette question, voir Pierre Martino, L'Orient dans la littérature française au XVIle et au XVIIIe siècle (Paris: Hachette, 1906), pp. 284-91; Maurice Roelens, in La Hontan, Dialogues (1973), p. 47. Enfin, rappelons que Geoffroy Atkinson signale aussi le rôle joué par le personnage d'Andrenio du Criticon, adaption espagnole de Havy ben Yaqhan d'Ibn Thofoël, écrit vers 1150, par Balthazar Gracian et traduit en français, sous le titre de L'Homme détrompé, ou le Criticon, 'où on trouve l'histoire d'un sauvage, initié, tel Vendredi, à l'humanité par un savant pédagogue, qui développe à son endroit une pédagogie évoquant par avance de mythe de la statue développé plus tard par Buffon, Charles Bonnet et Condillac': G. Gusdorf, La Révolution galiléenne (Paris: Payot, 1969), II, 281. Voir aussi G. Atkinson, The Extraordinary Voyage in French Literature from 1700 to 1720, (New York: Burt Franklin, 1967), pp. 31-33. Au reste, l'idée de promener un 'enfant de la nature' dans les salons de la société française pour l'amener à se 
prononcer avec naïveté sur celle-ci se trouve déjà dans l'Histoire de la Religion des Turcs de Michel Baudier (Paris: Cramoysie, 1625). Voir G. Atkinson, Les Relations de voyages du XVIIe siècle (Genève: Slatkine Reprints, 1972) pp. 41-43.

23 En ce sens, la société amérindienne apparaît comme 'un révélateur des tares et des vices de la société occidentale' et elle devient une 'référence' à partir de laquelle s'expriment les critiques que formulent les 'philosophes.' Voir Maurice Roelens, op. cit., 60.

24 Voir Nouveaux Voyages (1703) I, 51-56.

25 En fait, pour Michèle Duchet, la question est posée: 'Dans les livres,' écritelle, 'n'aimera-t-on par surtout ce qui crée l'illusion d'une présence: les discours, les harangues, les sentences, les traits de bravoure ou de cruauté, tout ce qui donne à l'homme sauvage une chance d'exister et d'entrer dans un monde de relations et d'échanges en marge de son propre monde et en marge du monde des civilisés?': op. cit., pp. 42-43.

26 Pour Maurice Roelens, La Hontan paraît être des plus 'représentatifs de cette première vague de philosophie critique, souvent plus radicale que la philosophie des Lumières qui a déterminé entre 1685 et 1715 ce qu'il est convenu d'appeler depuis Paul Hazard la crise de la conscience européenne et qui a fourni aux philosophes du milieu du siècle problèmes, arguments et méthodes': in La Hontan, Dialogues (1973), 7. C'est l'auteur qui souligne.

27 Voir son analyse des Dialogues, qu'il attribue à Gueudeville, in La Légende du bon Sauvage Contribution a l'étude des origines du Socialisme (Paris: Editions politiques, économiques et sociales, 1946), pp. 113-14. C'est l'auteur qui souligne.

28 Rappelons avec Charles-André Julien que 'c'est en 1558 que naquit le bon sauvage dans la Complainte contre Fortune de Ronsard, inspirée par les Singularitez de Thevet, publiées en 1557': Les Français en Amérique au XVIIe siècle (Paris: SEDES et CDU, 1976), p. 14.

29 Voir P. Berthiaume, Périples français en Amérique (Lille: Thèse d'État, 1987), pp. 421-24.

30 Le Grand Voyage dv Pays des Hurons (Paris: Denis Moreau, 1632), p. 65. Voir aussi pp. 293-95.

31 Op. cit. (1654), p. 396.

32 lbid., p. 397.

33 'Comme depuis la nature corrompuë par le peché de nos premiers peres, les loix ont esté absolument necessaires pour esclairer la raison, \& la faire marcher sans erreur dans les droits sentiers de la verité; il ne se faut pas estonner si la naissance, la vie \& les moeurs de nos Sauvages, qui sont privez de ces belles lumieres, ne sont remplies que de superstitions, d'erreurs \& de sottises, qui en donnant matiere de risée, tirent en mesme temps les larmes des yeux de ceux qui ont de veritables sentimens Chrestiens': Histoire generale des Isles (1654, p. 412).

34 Robert Lenoble, Esquisse d'une histoire de l'idée de nature (Paris: Albin Michel, $1969)$, p. 221. C'est l'auteur qui souligne. Voir aussi les pages 217-22 et 339-84.

35 Daniel Droixhe et Pol-Pierre Gossiaux, 'Avant-propos,' in L'Homme des lumières et la déconverte de l'Autre Etudes sur le XVIIe siècle, volume hors série 3 (Bruxelles, Editions de l'Université de Bruxelles, 1985), p. 8. Ce sont les auteurs qui soulignent. 
36 Ibid., p. 8. Ce sont les auteurs qui soulignent.

37 Sur cette question de la fracture entre la nature et la culture, voir Emile Bréhier, Histoire de la philosophie XVIIe-XVIIle siècle (Paris: P.U.F., 1985); Jean Ehrard, L'Idée de nature en France à l'aube des lumières, (Paris: Flammarion, 1970), pp. 275-311; Pol-Pierre Gossiaux, 'Séquences de l'histoire dans l'anthropologie des lumières Problèmes et mythes,' in Britta Rupp-Eisenreich, Histoires de l'anthropologie (XVIe-XIXe siècles) (Paris: Klincksieck, 1984), pp. 67-68; Pol-Pierre Gossiaux, 'Anthropologie des Lumières (culture "naturelle" et racisme rituel), ' in Daniel Droixhe et P.-P. Gossiaux, op. cit., pp. 49-69. Précisons que nous nous inspirons ici surtout des deux textes de Pol-Pierre Gossiaux.

38 Journal de la campagne des Isles de l'Amerique (1709), 182-83. Ce que confirment au reste leur physique et leurs qualités intellectuelles: ils sont 'bien proportionnés; gros, puissants, fort robustes, si dispos, si sains' qu'ils vivent cent ans, en outre, ils ont le 'raisonnement bon \& l'esprit aussi subtil que le peuvent avoir des personnes qui n'ont aucune teinture de lettres': ibid., pp. 184 et 185 .

39 Gautier du Tronchoy, op. cit., p. 185.

40 Par exemple, Gautier du Tronchoy note que les Amérindiens élèvent leurs enfants 'plutot en brutes qu'en hommes raisonnables, ne leur aprenant n'y [sic] civilitez n'y honneurs; ne sçachant ce que c'est que de remercier, de dire bon jour, bon soir, n'ont aucune honte de leur nuditez': Journal de la campagne des Isles de l'Amerique (1709), p. 193.

41 La Hontan, Dialogues (1704), p. 53. C'est l'auteur qui souligne.

42 'Je dis donc que ce que vous appelez argent, est le démon des démons, le tiran des François; la source des maux; la perte des ames \& le sepulcre des vivans': Dialogues (1704), p. 53.

43 La Hontan, Dialogues (1704), p. 34.

44 Ibid., p. 41.

45 Ibid., p. 36.

46 D'autant qu'on ne saurait 'connoître ce que le grand Esprit demande': Dialogue (1704), p. 32.

47 Voir Dialogues (1704), pp. 61-62. Il est intéressant de noter que le discours d'Adario se fonde toujours sur une forme de rationalité utopique qui suppose l'existence d'un bien absolu, d'une justice absolue, coiffés par la raison et reconnus par tous. Point de jeux de passions, de rapports de force ou de faits d'évolution dans ses théories abstraites. Curieusement cet homme de la nature paraît penser en termes idéalistes. Il est vrai que la notion de 'nature' est elle-même une idée assez éloignée de la réalité dans ces Dialogues bien philosophiques.

48 Inutile de développer une argumentation connue que Gilbert Chinard a du reste magistralement exposée. Voir L'Amérique et le rêve exotique dans la littérature française au XVIIe et au XVIIIe siècle (Genève: Slatkine Reprints, 1970), pp. 167-87.

49 Op. cit. (1769), III, pp. 28-29. 
50 Autre exemple, encore bien romanesque, celui du personnage d'Ador dans la Relation de divers voyages de Dralsé de Grand-Pierre, qui soutient que 'le Monde, en sortant des mains de Dieu estoit un theatre heureux, où regnoit la paix, la sagesse, la nature dans tous leu[r]s charmes; [mais que] les hommes en ont fait un théâtre affreux, où regnent la discorde, la folie, \& la cruauté dans tout leur desordre \& leur amertume' (op. cit. [1718], 70-71), et qui en tire les conséquences qui s'imposent, soit passer en Afrique et en Amérique pour aller 'y estudier les différens moyens que la nature \& les passions ... suggerent [aux Noirs et aux Amérindiens] pour parvenir à la felecité' (ibid., 75). Enfin, cas limite, et précisément fictif, l'Ingénu. Voir l'étude du conte du même nom par Jacques Van den Heuvel in Voltaire, Romans et contes (Paris: Gallimard, bib. de la Pléiade, 1979), pp. 966-81. Ironiquement, le conte finit par influer sur les relations de voyages et en 1777, Jean Bossu prête à un chef 'Akanças' une harangue qui ne déparerait pas le texte de Voltaire. Voir Nouveaux Voyages (1777), pp. 98-99. Rappelons aussi le paradoxe que signale Gilbert Chinard qui observe que c'est en pillant les Relations et les Lettres édifiantes des Jésuites que les philosophes ont trouvé des exemples et des armes pour saper les fondements métaphysiques de la religion de l'Etat. Voir L'Amérique et le rêve exotique, pp. 122-50.

51 De là une ambiguité certaine, par exemple chez La Hontan quı comprena moins le mode de pensée amérindien qu'il ne l'utilise à des fins polémiques. C'est du reste ce que dénonce Samuel Engel lorsqu'il reproche au baron de 'prête[r], on ne sçait à quels barbares du Canada, ses propres idées, qui sont encore trés éloignées d'être justes': Supplément à l'Encyclopédie (Amsterdam: M.M. Rey, 1776), I, 352).

52 Voir G. Chinard, L'Amérique et le rêve exotique pp. 221-44.

53 Voir Histoire de la Louisiane (1758), III, 238-39.

54 Voir op. cit., II, 30-33.

55 Plus précisément, les protagonistes constatent que le roi d'Espagne n'a pas vraiment fait châtier François de Tolède, pourtant responsable de rapines et de meurtres commis sur les Amérindiens, ses alliés. Voir Nouveaux Voyages (1768), II, 37.

56 Journal d'un voyage fait par ordre du Roi (1744), pp. 340-41.

57 Rappelons que le souci d'exhaustivité n'est pas l'apanage de Charlevoix et qu'il fait partie du mode de pensée du siècle des Lumières. Ainsi Frézier décrit-il lui aussi les 'indiens du Chily' en exposant leur foi, leur gouvernement, leurs guerres et leurs armes, leurs fêtes et leurs jeux, leur 'temperament \& nourriture,' leur aspect physique et leurs vêtements, leur habitation et leur société en plus de leurs méthodes agricoles et de leur commerce. Voir Relation du voyage de la mer du Sud aux côte du Chily (1716), pp. 52-70.

58 La Condamine ne peut s'empêcher de noter 'l'insensibilité' qui 'fait la côtes base' du caractère des Amérindiens de l'Amazonie qui 'passent leur vie sans penser' et qui demeurent dans une espèce d"enfance' durant toute leur existence, pour conclure qu'on 'ne peut voir sans humiliation combien l'homme abandonné à la simple nature, privé d'éducation \& de société, diffère peu de la bête': Relation abrégée (1745), pp. 52-53. Sur la question de la disparition des Amérindiens, voir Michèle Duchet, op. cit., pp. 149-63. 
59 À titre d'exemple, comme le rappelle Gilbert Bosetti au sujet des Amazones, elles se déplacent toujours plus loin au-delà des frontières du monde connu, de 'Zachibar' ou 'Canghibar,' l'île des femmes chez Marco Polo, à une région mal définie entre la Chaldée et l'Ethiopie chez Jean de Mandeville, puis en Amazonie Américaine. Voir 'Marco Polo et l'horizon austral de l'océan indien,' in Le Voyage austral (Grenoble: ELLUG, 1984), pp. 13 et 20.

60 Lapérouse, Voyage (1985), II, 147.

61 Les Tlingits paraissent féroces, fourbes, vindicatifs, cruels et voleurs à Lapérouse. Voir Voyage (1985), II, 147. Ailleurs, il écrit: 'll n'y a personne qui ayant lû les relations des derniers voyageurs, puisse prendre les Indiens de la Mer du S. pour des sauvages; ils ont au contraire fait de trèz grands progrèz dans la civilisation, et je les crois aussy corrompûs qu'ils peuvent l'être, relativement aux circonstances ou ils se trouvent' (ibid., II, 75). Bien sûr, toute la remarque est empreinte d'ironie à l'endroit des thèses sur les 'bons Sauvages.' Texte cité: Voyage (1985), II, 155.

62 Ibid., II, 197.

63 Journal, in E. Taillemite, Bougainville et ses compagnons autour du monde (1977), II, 373.

64 On pense par exemple aux dernières lignes de la relation du prince de Nassau-Siegen qui s'écrie: 'mais c'est le siècle de l'humanité et il faut du moins esperer que les Européens ne veulent plus faire connoissance avec leurs frères de l'hémisphère austral que pour leur enseigner la Vérité, et les rendre heureux': Journal, in E. Taillemite, Bougainville et ses compagnons autour du monde (1977), II, 415. Toutefois, c'est surtout l'expédition de Lapérouse qui constitue un cas exemplaire: 'de tous les bienfaits que la munificence du roi veut faire présent aux habitants des pays nouvellement découverts,' expliquent les instructions, 'les végétaux utiles à la nourriture des hommes sont sans contredit ceux qui leur procureront les biens les plus durables et les plus propres à augmenter leur bonheur': Lapérouse, Voyage (1797), I, 15. De fait, la quatrième partie du mémoire du roi, intitulée 'De la conduite à tenir avec les naturels des pays où les deux frégates pourront aborder,' illustre on ne peut mieux l'humanisme de la 'généreuse et tragique expédition.'

D'ailleurs, au dire de Lapérouse, le projet de la campagne est essentiellement humaniste: 'heureusement pour ces peuples, qu'ils ont été connûs à l'époque ou la religion ne sert plus de prétexte aux violences, et à la cupidité. Les navigateurs modernes n'ont pour objet en decrivant les moeurs des peuples nouveaux que de complêter l'histoire de l'homme': Voyage (1985), II, 99-101. À titre d'exemple, à l'île de Pâques, on débarqua 'des chêvres, des brebis, des cochons' et on planta 'des graines d'oranger, de citronier, de coton, de maïs, et généralement touttes les especes qui pouvaient reüssir' dans l'île: Voyage (1985), II, 67. Il est vrai que le geste n'est pas absolument désintéresse. Dans son Mémoire phisiologique et pathologique sur les Americains, Rollin observe que ces dons pourront aussi 'assurer aux voyageurs, qui lui succéderont, des rafraichissements de toutes especes' (A.N., Marine $3 \mathrm{JJ} 387$, pièce 5. F.90).

65 Journal, in E. Taillemite, Bougainville et ses compagnons autour du monde (1977), II, 375 . 
66 Bel exemple de cette conception dans les négociations entre la cour de France et celle de Madrid au sujet de la possession des Malouines. Voir J.-E. Martin-Allanic, Bougainville navigateur et les découvertes de son temps (Paris: P.U.F., 1964), I, 164-202 et 223-94.

67 Une nuance importante dans le cas de Lapérouse qui refuse de prendre possession de l'île de 'Mowee' en expliquant que les usages des Européens à cet égard lui paraissent inacceptables. Voir Voyage, 1985, II, 99-101. Toutefois, à l'île Sakhaline, il n'hésitera pas à donner à nombre de lieux des noms français, il est vrai, sans en prendre formellement possession.

68 Et qui explique encore le discours préliminaire de d'Alembert dans l'Encyclopédie.

69 Il fonde l'évolution de l'humanité sur l'agriculture, ce qui d'emblée exclut le Sauvage, le plus souvent nomade et chasseur: 'c'est l'Agriculture qui a conduit les hommes par la main, de degrés en degrés, de la constitution agreste à la constitution politique: plus un terrein est-il propre à être ensemencé, plus les graines comestibles y abondent, \& plus les possesseurs de ces champs fertiles \& de ces semences précieuses s'humanisent, s'ils s'adonnent à la culture, qui commencera par les rendre sédentaires, \& dès-lors ils sont à demi-policés': Recherches philosophiques sur les Américains (Berlin: s.é., 1771), I, 99 .

70 Dans le discours préliminaire, De Pauw, constate qu'une 'moitié' du globe, soit l'Amérique, est 'tellement disgraciée par la nature, que tout y [est] ou dégénéré, ou monstrueux; (op. cit., I, p. iii). Au reste, il explicite sa pensée en produisant un portrait de plus noir de l'Amérindien, énervé, hypocrite, lâche, vicieux, etc. Voir op. cit., I, 34-91 et II, 153-208. À titre d'exemple, rappelons ce qu'il écrit des chasseurs: 'errans \& incertains de leur sort d'un jour à l'autre, ils doivent craindre la réunion \& la multiplication de leurs semblables, comme le plus grand des malheurs; parce que le gibier, bien moins fécond que le poisson, se dépeuple dans tous les Pays du monde, à proportion que le nombre d'hommes croît. Un Sauvage chasseur cherche les solitudes, s'écarte autant qu'il peut de toute habitation humaine, \& s'éloigne à chaque pas de la vie sociale: s'il construit une hutte, c'est plutôt pour s'y retirer que pour y être logé. Jamais en paix avec les hommes ou avec les animaux, son instinct est féroce \& ses mœurs barbares: plus son génie s'occupe-t-il des moyens de subsister, moins réfléchit-il sur la possibilité de se policer. Il est dans le genre-humain ce que sont les bêtes carnassières entre les quadrupèdes, insociable' (op. cit., I, 101). Déjà en 1736, les vers du Mondain dénonçaient la figure du bon Sauvage, mais l'insolence et le caractère caricatural de la diatribe lui enlevaient une partie de sa valeur critique.

71 Antoine-Joseph Pernety, Dissertion sur l'Amérique et les naturels de cette partie du monde, in de Pauw, Recherches philosophiques sur les Américains (Berlin: s.é., 1771), III; Pierre Poivre, De l'Amérique et les Américains, ou observations curieuses du philosophe La Douceur, Qui a parcouru cet Hémisphère pendant la derniere Guerre, en faisant le noble métier de tuer des Hommes sans les manger (Berlin: Samuel Pitra, 1772).

72 Seul le peuple élu a su conserver la Tradition alors que les autres peuples qui ont émigré de la terre sacrée participaient d'une histoire qui les éloignait doublement du message originel. 
73 Sur cette question, voir Pol-Pierre Gossiaux, 'Séquences de l'histoire dans l'anthropologie des lumières Problèmes et mythes,' in Britta Rupp Eisenreich, op. cit., pp. 73-74.

74 Barrère, Nouvelle Relation de la France equinoxiale (1743), p. 146.

75 Entre autres, Denis Diderot tire d'une remarque du Voyage autour du monde un long Supplément qui pose le problème de la liberté en des termes philosophiques qui ont finalement peu à voir avec l'expérience de Bougainville, surtout si l'on pense à celle, plus concrète et plus critique, du Journal de son voyage. Voir Gilbert Chinard, L'Amérique et le rêve exotique, pp. 374-85.

76 Thomas Hobbes, De Cive ou les fondements de la politique, traduction de Samuel Sorbière présentation par Raymond Polin (Paris: Publications de la Sorbonne, 1981), p. 87.

77 'Nous avons en ce siècle un exemple de ce que je dis chez les Américains; \& dans les âges passés nous en avons eu chez les autres nations, qui maintenant sont civilisées \& florissantes, mais qui alors étoient en petit nombre, sauvages, pauvres, hideuses, \& privées de ces ornemens \& de ces avantages que la paix \& la société apportent à ceux qui les cultivent': Thomas Hobbes, op. cit., p. 87.

78 'Je conclus donc, que la loi de nature commande les bonnes mours \& la vertu, en ce qu'elle ordonne d'embrasser les moyens de la paix, \& qu'à juste titre elle doit être nommée loi morale': ibid., p. 120.

79 Ibid., pp. 121-22.

80 'Que l'état de nature, c'est-à-dire, d'une liberté absolue, telle qu'est celle de ceux qui ne gouvernment point \& qui ne sont sous aucun gouvernement, soit une anarchie, \& un état de guerre \& d'hostilité; que les maximes par lesquelles on évite un si fâcheux état soient les loix de nature; qu'aucun état ne puisse point subsister sans une souveraine puissance; qu'il faille obéir absolument à ceux qui l'exercent, c'est-à-dire, en tout ce qui ne répugne point aux commandemens de Dieu, c'est ce qu'aux chapitres précédens j'ai démontré, ce me semble, assez clairement': ibid., pp. 271-72.

81 Raymond Polin, introduction in Thomas Hobbes, op. cit., p. 33.

82 'La plus grande \& la principale fin que se proposent les hommes, lors qu'ils s'unissent à une Communauté, \& se soumettent à un Gouvernement, c'est de conserver leurs Propriétez, pour la conservation desquelles bien des choses manquent dans l'estat de Nature': J. Locke, Du Gouvernement civil, Où l'on traitte de l'Origine, des Fondemens, de la Nature, du Pouvoir, $\mathcal{E}$ des Fins des Sociétez Politiques (Amsterdam: Abraham Wolfgang, 1691), p. 161.

83 François Hartog, op. cit., p. 28. À noter que nous pervertissons ici la notion telle qu'elle exposée chez François Hartog. Pour lui, le 'modèle absent' est par exemple le sacrifice grec, jamais présent dans le texte d'Hérodote, mais toujours sous-entendu dans le discours comme référence nécessaire pour concevoir le sacrifice tel que pratiqué par les Scythes. Par ce modèle absent, Hérodote peut s'interroger sur le sacrifice de l'Autre et imaginer ce qu'il est.

84 Dans un article récent, Pierre-François Moreau observe que la pensée au siècle classique semble fondée sur la 'dissociation du réel.' Voir 'Nature, culture, Histoire' in François Châtelet et Gérard Mairet, op. cit., tome III, pp. 31-56. 
85 John Locke, op. cit., 15-16.

86 Locke cite notamment des exemples empruntés à José de Acosta, op. cit., 130 et 139 .

87 Ibid., p. 107.

88 Ibid., p. 108.

89 Ibid., p. 111.

90 Ibid., p. 1.

91 John Locke,Two Treatises of Government: in the former, the false principles, and foundation of Sir Robert Filmer, and his followers, are detected and overthrown. The latter is an essay concerning the true original, extent, and the end of civil government (London: A. Churchill, 1690). Une première traduction française parait l'année suivante à Amsterdam.

92 Comme l'observe Gilbert Chinard, 'le Supplément va compléter la peinture que Jean-Jacques Rousseau avait simplement esquissée dans le Discours de l'inégalité. Plus exactement encore, Diderot va se servir de l'exemple de Tahiti pour vérifier, localiser et rendre plus réelle la reconstitution hypothétique des premiers temps qui se trouve dans le second Discours' in Diderot, Supplément au Voyage de Bougainville (Paris-Baltimore: Droz-The John Hopkins Press, 1935), p. 51. Mais il s'agit ici d'un cas limite: le Supplément forme le point d'aboutissement de la figure du bon Sauvage en l'articulant sur le concept d'homme primitif. 'Pour dire les choses brutalement - et Diderot est là-dessus assez explicite dans la dernière partie du Supplément - le monde sauvage est mort à jamais; en même temps il nous appartient et il nous est impossible de reprendre l'héritage de la "jeunesse du monde"': Georges Benrekessa, Le Concentrique et l'excentrique: marges des lumières (Paris: Payot, 1980), p. 222. Voir aussi aux pages 213-24. Essentiellement, la démonstration que réalise Diderot vise à montrer la dénaturation de l'homme par des lois qui ne se règlent par sur les exigences de la nature. Dans le discours qui oppose un Tahitien à un prêtre, le premier montre que les lois, inventées par l'homme, vont à l'encontre de ses besoin élémentaires. De là des conflits insolubles: 'où en seraistu réduit, si tes trois maîtres, peu d'accord entre eux, s'avisaient de te per mettre, de t'enjoindre et de te défendre la même chose, comme je pense qu'il arrive souvent? [demande le primitif] Alors pour plaire au Prêtre, il faudra que tu te brouilles avec le Magistrat; pour satisfaire le Magistrat, il faudra que tu mécomptes le grand Ouvrier, il faudra que tu renonces à la Nature. Et sais-tu ce qui en arrivera? c'est que tu les mépriseras tous les trois, et que tu ne seras ni homme, ni citoyen, ni pieux, que tu ne seras rien': D. Diderot, op. cit., 142-43. De là aussi la conclusion attendue: 'attachetoi à la nature des choses et des actions, à tes rapports avec ton semblable, à l'influence de ta conduite sur ton utilité particulière et le bien général' (ibid., 143). Au total, pour Diderot, les moeurs se définissent comme 'une conduite conséquente à des Lois bonnes ou mauvaises. Si Les sont bonnes, les moeurs sont bonnes; si les Lois sont mauvaises, les moeurs sont mauvaises. Si les Lois bonnes ou mauvaises, ne sont point observées, la pire condition d'une société, il n'y a point de moeurs' (ibid., p. 179). Voir aussi Michèle Duchet, op. cit., pp. 345-405.

93 Le tout au nom d'une dénonciation de la notion de propriété: au départ, l'homme 'est innocent et doux partout où rien ne trouble son repos sa 
sécurité. Toute guerre naît d'une prétention commune à la même propriéte' (D. Diderot, op. cit. 111). C'est pourquoi Diderot conclut ainsi: 'Voulez-vous savoir l'histoire abrégée de presque toute notre misère? La voici. Il existait un homme naturel; on a introduit au dedans de cet homme un homme artificiel, et il s'est élevé dans la caverne une guerre continuelle qui dure toute la vie' (Ibid., 190). Bien sûr, la démonstration repose sur le postulat de la bonté naturelle de l'homme et Diderot, tout comme Rousseau, du reste, fait peu de cas des pulsions agressives propres à l'être humain.

94 'Au sortir de mes mains,' soit celles de la nature, l'homme est 'simplement muni des facultés propres à recevoir' des idées métaphysiques et morales et il est 'plutôt indifférent à tout mouvement': Code de la nature (Paris: Gilbert Chinard, 1950). Il est intéressant aussi de constater que pour Morelly les moeurs des Sauvages ne sont pas celles de la nature, mais le résultat d'un dérèglement occasionné par une mauvaise interprétation des lois de la nature car celle-ci, à ses yeux, ne produit que des 'inclinaisons paisibles' (ibid., 184).

95 À noter qu'on trouve le même recours au concept chez ceux qui entendent, au contraire de Diderot et de Rousseau, démontrer la valeur de la socialisation de l'humanité, notamment chez l'auteur anonyme des Observations sur les Sauvages \& l'Amérique, publiées dans les numéros du Journal encyclopédique du 15 août et du ler septembre 1765, qui demande: si l'homme 'eut été destiné à vivre solitaire comme les autres animaux carnassiers, auroit-il pu contredire la loi de la nature jusqu'à vivre en société?' Journal Encyclopédique, 15 août $1765,111$.

96 Jean-Jacques Rousseau, Discours sur l'origine et les fondements de l'inégalité parmi les hommes (Paris: Aubier Montaigne, 1973), p. 56. À noter que Rousseau paraît ici influencé par Sénèque dans son rejet de la culture et de la civilisation, sinon par la pensée protestante. Voir G. Pire, Annales Jean-Jacques Rousseau, XXXIII, 57-92, et Angèle Kremer-Marietti, introduction au Discours, pp. 11-15.

97 'Laissant donc tous les livres scientifiques qui ne nous apprennent qu'à voir les hommes tels qu'ils sont faits, et méditant sur les premières et plus simples opérations de l'âme humaine, j'y crois apercevoir deux principes antérieurs à la raison, dont l'un nous intéresse ardemment à notre bien-être et à la conservation de nous-mêmes, et l'autre nous inspire une répugnance naturelle à voir périr ou souffrir tout être sensible, et principalement nos semblables' (op. cit., pp. 58-59).

98 Michèle Duchet, op. cit, p. 272. C'est l'auteur qui souligne.

99 Jean-Jacques Rousseau, op. cit., pp. 62-63.

100 Michèle Duchet, op. cit., p. 279. C'est l'auteur qui souligne.

101 Henri Gouhier, 'Nature et histoire dans la pensée de Jean-Jacques Rousseau,' in Annales de la Société Jean-Jacques Rousseau, XXXIII (1953-1955), 11. Angèle Kremer-Marietti: 'Ainsi la fiction scientifique de l'état de nature est une véritable hypothèse de travail montrant que l'homme est un produit social qui, dans un état de dispersion et d'isolement, est un être proche de l'animalité, mais privé des caractères définis des différentes espèces. Ce que Rousseau tente, c'est moins de nous enseigner ce que fut l'état de nature, qui selon Rousseau lui-même n'exista probablement jamais, mais de nous faire penser que, dépouillé des caractères historiques et sociaux, l'homme ne peut 
se définir que dans un état d'indifférence morale comme un être ni bon ni méchant, dans un état d'équilibre et d'auto-suffisance proche d'une sagesse insouciante dénuée de passions et privée d'évolution' (in Rousseau, op. cit., p. 26-27). Voir aussi E. Durkeim, Montesquieu et Rousseau précurseurs de la sociologie (Paris: M. Rivière, 1966), pp. 115-20.

102 En effet, dans Leviathan, Hobbes observe au sujet de l'état de nature: 'there is no place for Industry; because the fruit thereof is uncertain: and consequently no Culture of the Earth, no Navigation, nor use of the commodities that may be imported by Sea; no commodious Building; no Instruments of moving, and removing such things as require much force; no Knowledge of the face of the Earth; no account of Time; no Arts; no Letters; no Society; and which is worst of all, continuall feare and danger of violent death; And the life of man, solitary, poore, nasty, brutish, and short': op. cit., (LondonNew York: Dent \& Dutton, 1970), pp. 64-65. Certes, c'est la vie de l'homme qui est solitaire, et non chaque individu, mais l'idée a pu séduire Rousseau.

103 Jean-Jacques Rousseau, op. cit., p. 100. Sur ce sujet, voir l'excellent tableau chronologique des différents états de l'homme chez Rousseau in Michèle Duchet, op. cit., p. 313.

104 Michèle Duchet, op. cit. p. 292. L'auteur fait allusion à l'Essai sur l'origine des langues.

105 Jean-Jacques Rousseau, op. cit. p. 65. Voir aussi les Confessions, livre VIII et plus largement, Michèle Duchet, op. cit., pp. 265-313.

106 De fait le concept d'état de pure nature paraît bien reposer sur une documentation extraite des relations des voyageurs. C'est du moins ce que montre G. Pire dans un article intitulé 'Jean-Jacques Rousseau et les relations de voyages,' in R.H.L.F., 56e année, no. 3 (juillet-sept. 1956), 355-78. Sur cette question, voir aussi Emile Bréhier, op. cit, pp. 415-20.

107 Jean-Jacques Rousseau, op. cit., p. 89.

108 Ibid., p. 101.

109 Ibid., p. 69.

110 Condillac, Etienne Bonnot de, Traité des sensations, à Madame la Comtesse de Vassé (Paris: de Bure l'aîné, 1754); Denis Diderot, Supplément au voyage de Bougainville (1935); Joseph-Marie Gros de Besplas, De l'utilité des voyages relativement aux sciences et aux moeurs (Paris: Berthier, 1763); D'Holbach, Paul Henri-Dietrich, baron, Systême de la nature; ou des Loix du monde physique $\mathcal{E} d u$ monde moral, par M. Mirabaud (Londres: s.é., 1770); Morelly, Code de la nature ou le véritable esprit de ses lois (Paris: Gilbert Chinard, 1950); Jean-Jacques Rousseau, Discours sur l'origine et les fondemens de l'inégalité (1755). Au sujet de l'anthropologie des Buffon, Voltaire, Diderot et Rousseau, voir Michèle Duchet, Anthropologie et Histoire au siècle des Lumières (Paris: Flammarion, 1977).

111 Il suffit de comparer son 'Journal' de mer au récrit publié pour s'en convaincre.

112 Le phénomène n'est pas nouveau: 'il convient néanmoins, de se rappeler,' écrit G. Atkinson au sujet des voyageurs au XVIIe siècle, qu'ils 'étaient, pour la plupart, nourris de Platon, de Thomas Morus, ou de Bacon. Ils étaient donc préparés, avant de voyager, à trouver des 'républiques' dans des pays 
sauvages ou demi-civilisés. Il y a plus. Le public, qui lisait des voyages, demandait des nouveautés. Celui qui ne voyait à l'étranger que des rois, des magistrats, des prêtres, inférieurs à ceux de l'Europe, était un auteur peu intéressant. Le public n'achetait des voyages que pour apprendre du nouveau' Les Relations de voyages au XVIIe siècle (1972), p. 47.

113 Journal de la campagne (1709), p. 88.

114 À ce sujet d'ailleurs, Gilbert Chinard a montré à quel point les missionnaires au XVIIe siècle n'ont pas toujours clairement perçu le danger d'exalter la nature apparemment innocente des Amérindiens. Voir L'Amérique et le rêve (1970), pp. 122-50.

115 Tout juste après avoir signalé le bonheur des Caraibes, Gautier du Tronchoy s'empresse de souligner leur répugnante idolâtrie. Ailleurs, au sujet des mêmes Caraibes, Thibault de Chanvalon observe: 'les infidélités, les trahisons, les parjures, les vols \& les assassinats, si communs chez les nations civilisées, leur sont inconnus. La religion, la morale, les loix, les échaffauds \& les supplices, ces digues élevées pour la défense de tous contre tous, \& qui ne peuvent arrêter parmi nous ces désordres, sont inutiles à ces hommes qui ne suivent que la nature; nos crimes leur font horreur': Voyage à la Martinique (1763), p. 56. C'est l'auteur qui souligne. Pourtant, à peine cinq pages plus haut, il écrivait au sujet de l'amour chez eux: 'jamais la moindre démonstration de tendresse ou d'amitié pour ce sexe si recherché par les nations policées, si avili par celles qui ne suivent que la nature' (ibid., 51). Si le discours dit la nostalgie d'un monde sans malédiction, il évacue vite l'angoisse qu'une telle proposition implique en la reniant en même temps qu'il la formule.

116 Michèle Duchet, Le Partage des savoirs (Paris: Editions La Découverte, 1985), p. 213.

117 À noter que ce 'délire' n'est pas pratiqué par tous. Sans rappeler ce qu'écrit Voltaire au sujet de 'la pure nature' dans Candide, on peut citer les vers d'un voyageur... de cabinet:

On nous a trop vanté l'homme de la nature,

Nul être plus que lui n'a besoin de culture,

Il doit apprendre tout jusqu'à l'art d'être heureux.

Quoiqu'en dise Rousseau que j'aime \& que j'honore,

Si l'homme en nos cités n'est pas exempt de maux,

Le Sauvage est cent fois plus misérable encore.

Et vices, \& malheurs, il a tous les fléaux;

Sans plaisirs, sans vertus, sans devoirs qu'il respecte,

Son coeur est aussi vil que sa vie est abjecte.

C'est la société dont les heureux travaux

De la nature à l'homme ont assuré l'empire;

C'est elle qui lui montre à penser, à sentir;

De ses désirs fougueux prêts à l'assujettir,

C'est elle qui l'instruit à craindre le délire.

Louis-Gabriel Bourdon, Voyage d'Amérique, dialogue en vers entre l'Auteur $\mathcal{E}$ l'abbé *** (Londres et Paris: Pichard, 1786), pp. 9-10. 
118 Prévost, Antoine, Histoire du chevalier Des Grieux et de Manon Lescaut, in Oeuvres de Prévost (Grenoble, 1976), tome I, 432.

119 Les Malheurs de l'inconstance (Paris: Desjonquères, 1983), pp. 316-17.

120 Voyage dans les forêts de la Guyane française (1853), pp. 16-17. Il est d'ailleurs intéressant de constater que pour Malouet les Amérindiens vivent 'véritablement dans un état de société' (Ibid., 62). Ce que perd la figure de l'Autre, la nature le récupère. Rappelons aussi que déjà en 1758, Le Page du Pratz définissait un rapport avec la nature qui annonce celui qu'on trouve chez Malouet: 'j'étois fortement occupé de la beauté des Pays que j'avois vûs,' écrit-il au retour d'une expédition en forêt, 'j'aurois désiré finir mes jours dans ces charmantes Solitudes, éloigné du tumulte du monde, de l'avarice \& de la fourberie: c'est là, disois-je en moi-même, que l'on goûte mille plaisirs innocens, \& qui se répetent avec une satisfaction toujours nouvelle: c'est là que l'on est exempt de la critique, de la médisance \& de la calomnie; c'est dans ces riantes Prairies qui s'étendent souvent à perte de vûe, \& où l'on voit tant de différentes espèces d'animaux, que l'on a lieu d'admirer les bienfaits du Créateur; c'est là enfin, qu'au doux murmure d'une eau pure \& vive; c'est là disois-je, qu'enchanté des concerts des oiseaux qui remplissent les bosquets voisins, l'on peut contempler agréablement les merveilles de la Nature \& les examiner à loisir': Histoire de la Louisiane (1758), I, 263-64.

121 Bien sûr, se dessine ici la sensibilité d'un Chateaubriand qui écrira quelques années plus tard. 